\title{
Comment on "Analyses of Mössbauer Experiments in a Rotating System: Proper and Improper Approaches" [Annals of Physics Volume 418, July 2020, 168191]
}

\author{
Jaykov Foukzon \\ Center for Mathematical Sciences, Israel Institute of Technology, Haifa, Israel \\ Email: jaykovfoukzon@gmail.com
}

How to cite this paper: Foukzon, J. (2021) Comment on "Analyses of Mössbauer Experiments in a Rotating System: Proper and Improper Approaches" [Annals of Physics Volume 418, July 2020, 168191]. Journal of High Energy Physics, Gravitation and Cosmology, 7, 416-444.

https://doi.org/10.4236/jhepgc.2021.72024

Received: December 18, 2020

Accepted: March 31, 2021

Published: April 2, 2021

Copyright $\odot 2021$ by author(s) and Scientific Research Publishing Inc. This work is licensed under the Creative Commons Attribution International License (CC BY 4.0).

http://creativecommons.org/licenses/by/4.0/

\begin{abstract}
The notion of classical well localized trajectories of a single photon in Minkowski spacetime does not make any rigorous sense by the well-known existence of a proof that single photons cannot be well localized. This leads to principal difficultness when photodetection probability on relativistic non inertial frame of reference is considered. In order to resolve this tension, we extend canonical Minkowski geometry up to relevant point-free Minkowski geometry [Ann. Physics 423 (2020) 168329]. The photodetection probability density on uniformly rotating frame endrowed with point-free Lorentzian geometry is obtained. The result of S. A. Podosenov et al. [Ann. Physics 413 (2020) 168047] is obtained without any reference to unphysical notion of the classical trajectories of photon. The paper again shows the correctness of the remarkable result of Prof. C. Corda concerning the Mössbauer rotor experiment as new proof of general relativity, which has been awarded by the Gravity Research Foundation. In addition, the paper also shows various very elementary mistakes, misunderstandings and flaws by the self-called "YARK group", which is a group of fringe researchers who attempts to promote wrong science, in particular, against the relativity theory.
\end{abstract}

\section{Keywords}

Mössbauer Rotor Experiment, Uniformly Rotating Frame, Photodetection Probability Density, Minkowski Point-Free Geometry

\section{Introduction}

In paper [1] published recently A. L. Kholmetskii et al. argued that S. A. Podo- 
senov et al. paper [2] wrong and cannot explain novel Mössbauer experiment in a rotating system.

However, paper [1] contains a lot of principal mistakes: (i) First notice that [1] based on rejection the Einstein equivalence principle (EEP) (see [3]) and misconception in basic notion of GRT. By using these misconceptions A. L. Kholmetskii et al. argued that: "the problem of the physical interpretation of the observed energy shift between emission and absorption lines in a rotating system under the framework of general relativity open."

Of course after rejection the EEP under the framework of general relativity one explains nothing.

(ii) Secondary note that paper [1] based on misleading in quantum optics.

In quantum optics we dealing with the probability density $w(t, r), r \in \mathbb{R}^{3}$ such that $w(t,\|\boldsymbol{r}\|, \Omega) \delta \Omega \mathrm{d} t$ is the probability of the photon registration near point $\overrightarrow{\boldsymbol{r}}$, between instant $t$ and $t+\mathrm{d} t$ by a detector with angular size $\delta \Omega \ll 1$ sr. and located at the point $r \in \mathbb{R}^{3}$. The probability density $w(t, \boldsymbol{r})$ is not highly localized in free space except unphysical $1 \mathrm{D}$ case known from literature (see [4], 5B 1.2). Single photons cannot be localized by using photodetector. Nevertheless in order to disprove S. A. Podosenov et al. paper [2], A. L. Kholmetskii et al. in [1] naively argued that $\gamma$-quanta is a point particle and propagate along highly localized classical trajectory. But this is a missconcept.

Remark 1. But more importantly, these authors deliberately mislead readers by reporting absolutely false information about the experimental results stated in the classical papers [5] [6] [7] [8] [9].

A. L.Kholmetskii et al. wrote (see [1] p.5): However, "it becomes obvious that Podosenov et al. [2] did not even realize the fact that Equation (11) indicates a red shift of the frequency of the resonant radiation (i.e., $v_{R}<v_{0}$ ), whereas the equality $k=2 / 3$ in Equation (4), obtained in the experiments [9] [10] [11] [12] corresponds to the blue shift of the resonant radiation when $v_{R}>v_{0}$. We add that the same blue shift of the frequency of the resonant $\gamma$-quanta has been obtained in all other Mössbauer rotor experiments [1]-[6] (see corresponding ref. [5] [6] [7] [8] [9] in this paper) in the configuration where the source of resonant radiation was located on the rotational axis, and the resonant absorber was mounted on the rotor rim".

Remark 1. Note that in classical Mössbauer rotor experiments [1]-[6] only a red shift in full accordance with GRT prediction without any doubt were obtained.

Remark 2. (i) Note that nowel Mössbauer experiment in a rotating system is not the same as fundamental Kündig experiment but only essentially simplified version of Kündig experiment [9].

(ii) A. L.Kholmetskii et al. wrote (see [1] p.3):

"In these experiments, we did not repeat the approach by Kündig, who based himself on a linear Doppler modulation of the energy of the emitted resonant $\gamma$-quanta, because some unaccounted-for systematic errors in the 
evaluation of the coefficient $k$ in Equation (4) do inevitably emerge (see, e.g. [10]). Thus, we did not try to repeat directly the measurement scheme by Kündig, but followed the experimental scheme used in [1] [2] [3] [4] [6], where the source of resonant radiation is rigidly fixed on the rotor axis".

(iii) Note that in this simplified version the sign of the energy shift is not measured.

(iv) In Kündig experiment the energy shift is measured sucussesefully. The Equation (3) describes the Kündig's experimental data. For instance, if $R_{a}>R_{e}$, the energy that a photon must have for being absorbed by the absorber is smaller than the energy of the photon emitted by the emitter. In this case, for restoring the resonance condition, the absorber must be moved away from the emitter, thus compensating by first order Doppler effect the energy mismatch.

1) A. L. Kholmetskii et al. wrote (see [1] p.1): "As is known, the first series of Mössbauer rotor experiments aiming to verify the relativistic time dilation under laboratory conditions had been carried out in the early $60 \mathrm{~s}$ soon after the discovery of the Mössbauer effect (see, e.g., [1]-[6]). The latter effect leads to the following prediction for the relative energy shift between the line of a source of resonant radiation (located on the rotational axis) and the line of a resonant absorber (located at the rotor rim):

$$
\frac{E_{s}-E_{a}}{E_{s}}=-\frac{1}{2} \frac{u^{2}}{c^{2}}
$$

Here, $E_{s}$ is the energy of the resonant radiation for the source, and $E_{a}$ is the energy of the resonant radiation for the absorber, with $u$ standing for the tangential velocity of the absorber, and $c$ for the light velocity in vacuum (see Figure 1). We would like to point out that the "minus" sign on the rhs of Equation (1) corresponds to the blue shift of the energy of the resonant radiation, where $E_{a}>E_{s}$.

2) A. L. Kholmetskii et al. wrote (see [1] p.3): "Putting aside the deviation of Equation (3) from Equation (2), we emphasize that the estimation (3) anyway substantially disagreed with the classical relativistic prediction given by Equation (1), and due to this reason, it attracted considerable attention from the scientific community, indicating the need to carry out new Mössbauer experiments in a rotating system".

Remark 3. (i) Note that prediction (1) for the case of the Mössbauer rotor experiments wrong and based on complete rejection of the Einstein equivalence principle.

(ii) The characteristic resonance absorption frequency of the moving absorber at the rim should decrease due to time dilation, so the transmission of gamma rays through the absorber increases, which is subsequently measured by the stationary counter beyond the absorber. The maximal deviation from time dilation was $10^{-5}$. Such experiments were performed by Hay et al. [5], Champeney et al. [6] [7] and by Kündig [9]. 
Remark 4. Note that well known that commonly accepted true classical relativistic prediction is based on general relativity treatment and in contrary with wrong Equation (1) reads (see [4] chapt. 12, sect. 10) .

$$
\frac{E_{s}-E_{a}}{E_{s}}=\frac{1}{2} \frac{u^{2}}{c^{2}}
$$

where "plus" sign on the rhs of Equation (2) corresponds to the red shift of the energy of the resonant radiation, where $E_{a}<E_{s}$.

In Kündig paper [9] the Equation (2) presented in the following form:

$$
\frac{E_{a}-E_{s}}{E_{s}} \simeq-\frac{1}{2} \frac{\omega^{2} R_{a}^{2}}{c^{2}}=-\frac{1}{2} \frac{u^{2}}{c^{2}} .
$$

where "minus" sign on the rhs of Equation (1) corresponds to the red shift of the energy of the resonant radiation, where $E_{a}<E_{s}$, and where Mossbauer source is placed in the center of a system rotating with the angular velocity $\omega$, an absorber is mounted at a radius $R_{a}$, and a counter is at rest beyond the absorber.

Kündig wrote (see [9] p.1) "However, when the experiment is analyzed in a reference frame $\mathrm{K}$ attached to the accelerated absorber, the problem could be treated by the principle of equivalence and the general theory of relativity. The centrifugal force acting on the absorber is then interpreted as a gravitational force with the potential

$$
\Phi=-\frac{1}{2} R_{a}^{2} \omega^{2} .
$$

Thus, the observer in $\mathrm{K}$ will come to the conclusion that his clock is slowed down by the gravitational potential. The frequency $v_{a}$ measured in his frame of reference is given to a first approximation by

$$
v_{a}=v_{s}\left(1+2 \Phi / c^{2}\right)^{1 / 2} \simeq v_{s}\left(1+\Phi / c^{2}\right) .
$$

The fractional energy shift is

$$
\left(E_{a}-E_{s}\right) / E_{s} \simeq-\frac{1}{2 c^{2}} R_{a}^{2} \omega^{2} .
$$

Remark 5. We would like to point out that the "minus" sign on the rhs of Equation (3) corresponds to the red shift of the energy of the resonant radiation, where $E_{a}<E_{s}$ in accordance with true classical relativistic prediction is based on general relativity treatment [10] [11].

\section{Wrong Theoretical Descriptions of the Mössbauer Experiment in a Rotating System}

Note that wrong prediction is given by Equation (1) and named by A. L. Kholmetskii et al. the "classical" relativistic prediction is a classical mistake based on misunderstood what we really measured using rotating absorber of $\gamma$-quanta located on the rotor rim.

There exist a lot authors which naively treated Mössbauer experiment in a rotating system mistakenly using formula (4) for Doppler frequency (energy) shift 
it follows the frequency of absorbed radiation $v_{a}$ reads as [12]

$$
v_{a}=\frac{v_{0} \sqrt{1-u_{A}^{2} / c^{2}}}{\sqrt{1-u_{B}^{2} / c^{2}}},
$$

where $v_{0}$ is the proper frequency of gamma-quanta, $u_{A}$ is the velocity of point $\mathrm{A}$ at the emission moment, and $u_{B}$ is the velocity of point $\mathrm{B}$ at the absorption moment, see Figure 1.

From Equation (4) finally one obtains (see [8])

$$
\frac{v_{0}-v_{a b}}{v_{0}}=1-\frac{1}{\sqrt{1-u_{B}^{2} / c^{2}}} \simeq-\frac{u_{B}^{2}}{2 c^{2}}
$$

or in the following equivalent form related to Kündig notations

$$
\frac{E_{a}-E_{s}}{E_{s}}=\frac{v_{a}-v_{s}}{v_{s}}=\frac{\omega^{2}}{2 c^{2}}\left(R_{a}^{2}-R_{e}^{2}\right) .
$$

There exists apparent contradiction between Equation (9) and Equation (6) from Kündig paper [9].

Remark 6. (i) Note that a "proof" of the Equation (8) from T. Yarman et al. [12] wrong since this "proof" implicitly uses a postulate named in literature "Hypothesis of Locality".

(ii) Remind that the Hypothesis of Locality [13] [14] [15] is tacitly assumed that: any accelerated observer measures the same physical results as a standard inertial observer that has the same position and velocity at the time of measurement. For practical purposes, the hypothesis of locality replaces the accelerated observer by an infinite sequence of otherwise identical momentarily comoving inertial observers. Every inertial observer is endowed with a natural orthonormal tetrad frame in Minkowski spacetime. Therefore, the same holds for an accelerated

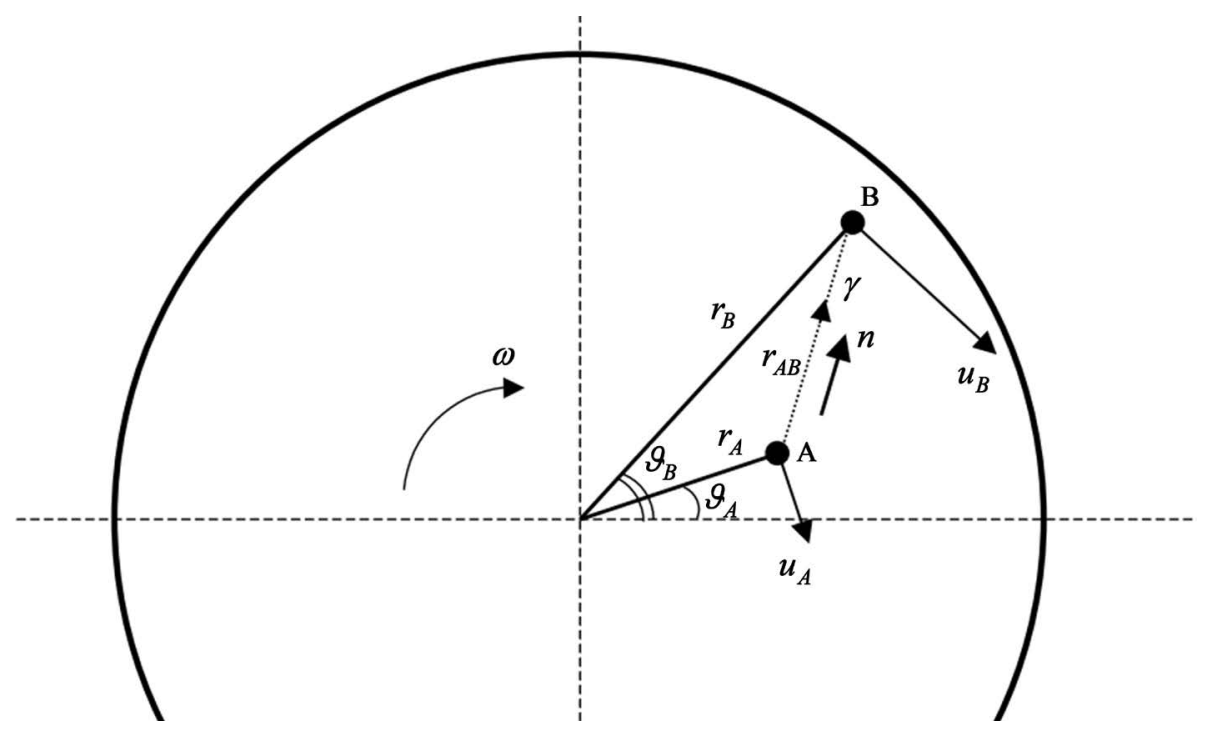

Figure 1. Adopted from [12]. Diagram for calculation of the Doppler effect in a rotating system between a point-like emitter (located in the point $\mathbf{A}$ at the emittance moment) and point-like receiver (located in the point $\mathbf{B}$ at the receiving moment). 
observer by the hypothesis of locality.

(iii) A restricted hypothesis of locality is the so-called clock hypothesis, which is a hypothesis of locality only concerned about the measurement of time. This hypothesis implies that a standard clock in fact measures $\tau, \mathrm{d} \tau=\sqrt{1-\beta^{2}(t)} \mathrm{d} t$, along its path; is then the proper time along this accelerated path.

According to most experiments, the hypothesis of locality seems to be true. No experiment has yet shown the hypothesis of locality to be violated (outside of radiation effects).

Remark 7. Note that for the radiation effects in rotating frame the Hypothesis of Locality obviously wrong since Hypothesis of Locality contradicts with EEP, it follows from consideration below.

Note that the energy of a particle of mass $m$ at rest in a constant gravitational field is given by [10] [11]: $E=m c^{2} \sqrt{1+\Phi / c^{2}}$ where $\Phi$ is the newtonian gravitational potential. If the particle is a nucleus in an exited state, one obtains

$$
E=\left(m c^{2}+\Delta E\right) \sqrt{1+\Phi / c^{2}},
$$

where $\Delta E$ is the energy difference between the two levels of the nuclear transition. Then, the energy difference between the two levels of the nuclear transition is modified by the gravitational potential by the multiplier $\sqrt{1+\Phi / c^{2}}$. Thus the angular frequency of the nuclear transition is given by: $v(\Phi)=v_{*} \sqrt{1+\Phi / c^{2}}$, where $v_{*}$ being the transition frequency without gravitational field [10] [11]. According to the weak equivalence principle, an acceleration field is locally indistinguishable from a gravitational one. Then, in a reference frame corotating with the rotor, the energy of a photon emitted by the source without recoil is given by:

$$
E_{s}=\Delta E \sqrt{1-\omega^{2} R_{s}^{2} / c^{2}},
$$

since $\Phi=-1 / 2 \omega^{2} R_{s}^{2}$ is the pseudo-gravitational potential due to acceleration. Analogously, the energy of the photon that can be absorbed by the absorber is given by:

$$
E_{a}=\Delta E \sqrt{1-\omega^{2} R_{a}^{2} / c^{2}}
$$

Therefore

$$
\frac{E_{a}}{E_{s}}=\frac{\sqrt{1-\omega^{2} R_{a}^{2} / c^{2}}}{\sqrt{1-\omega^{2} R_{s}^{2} / c^{2}}} .
$$

From Equation (13) in the approximation of small welocities $\omega R_{a} \ll c$ one obtains

$$
\frac{E_{a}-E_{s}}{E_{s}}=\frac{1}{2} \frac{\omega^{2}}{c^{2}}\left(R_{s}^{2}-R_{a}^{2}\right) .
$$

Remark 8. The cause of the violetion of the Hypothesis of Locality for the case of the radiation effects in rotating frame clear from Equation (12). 


\section{Non Highly Localizability of the Probability Density Corresponding to One-Photon State in 2D Space Dimension}

Remind that in quantum optics we dealing with the probability density $w(t, \boldsymbol{r}), \boldsymbol{r} \in \mathbb{R}^{3}$ such that $w(t,\|\boldsymbol{r}\|, \Omega) \delta \Omega \mathrm{d} t$ is the probability of the photon registration near point $\boldsymbol{r}$, between instant $t$ and $t+\mathrm{d} t$ by a detector with angular size $\delta \Omega \ll 1$ sr and located at the point $r \in \mathbb{R}^{3}$. The probability density $w(t, \boldsymbol{r})$ is not highly localized in free space except unphysical $1 \mathrm{D}$ case known from literature (see [4], 5B 1.2). Although below we will use the concept of the photon position vector $\boldsymbol{r}$, we will keep in mind that in fact this is the position of the photon detector. We consider 2D space dimension wave packet with $\boldsymbol{r} \in \mathbb{R}^{2}$, but without los of generality. Let us consider a one-photon state of the form (see [4], complement 5B).

$$
|1\rangle=\sum_{l} c_{l}\left|0, \cdots, n_{l}=1,0, \cdots\right\rangle=\sum_{l} c_{l}\left|1_{l}\right\rangle,
$$

where

$$
\sum_{l}\left|c_{l}\right|^{2}=1
$$

It changes in time and the state is given at time $t$ by

$$
|1(t)\rangle=\sum_{l} c_{l} \exp \left(-i \omega_{l} \times t\right)\left|1_{l}\right\rangle .
$$

The photodetection signal at time $t$ and fixed point $\boldsymbol{r}=(r, \varphi), r=\|\boldsymbol{r}\|$ (see Figure 1) is given by [4]:

$$
w(\boldsymbol{r}, t)=w(\|\boldsymbol{r}\|, \varphi, t)=s \| \hat{\boldsymbol{E}}^{(+)}(\boldsymbol{r})|1(t)\rangle \|^{2}=s\left|E^{(+)}(\boldsymbol{r}, t)\right|^{2},
$$

where $\varphi$ is angle $(\boldsymbol{r}, \boldsymbol{X})$ between axis $\boldsymbol{X}$ and vector $\boldsymbol{r}$ (see Figure 1), $s$ is the sensitivity of detector and where

$$
E^{(+)}(\boldsymbol{r}, t)=\sum_{l} c_{l}\left(\boldsymbol{k}_{l}\right) \varepsilon_{l} \exp \left[i\left(\boldsymbol{k}_{l} \cdot \boldsymbol{r}-\omega_{l} \times t\right)\right],
$$

and

$$
\boldsymbol{k}_{l} \cdot \boldsymbol{r}=\left\|\boldsymbol{k}_{l}\right\| \times\|\boldsymbol{r}\| \times \cos \left(\boldsymbol{r}, \boldsymbol{k}_{l}\right)=\omega_{l} \times\|\boldsymbol{r}\| \times \cos (\varphi, \theta),
$$

see Figure 1.

We consider now a set of coefficients $c_{l}=c_{l}\left(\boldsymbol{k}_{l}\right)$ different from zero for values of $\boldsymbol{k}_{l}$ distributed over some bounded region $G_{\boldsymbol{k}_{0}}$ of $\boldsymbol{k}$-space of extent $\delta k_{x}, \delta k_{y}$ about a value $\boldsymbol{k}_{0}$.

We thus obtain, at time $t=0$, a $2 \mathrm{D}$ wave packet localized in a volume of $\boldsymbol{x}$-space with dimensions of the order of $\hbar\left(\delta k_{x}\right)^{-1}, \hbar\left(\delta k_{y}\right)^{-1}$. When the same set of coefficients $c_{l}=c_{l}\left(\boldsymbol{k}_{l}, \varphi\right)$ is substituted into (17), we thus obtain a photodetection signal (18) that differs from zero only within some bounded region $G_{x}$ of $\boldsymbol{x}$-space of extent $\hbar\left(\delta k_{x}\right)^{-1}, \hbar\left(\delta k_{y}\right)^{-1}$.

Remark 9. The volume of this region $G_{x}$ generally increases without limit as time goes by, and this in each space dimension. Therefore, there is now well localized classical trajectories of such $\gamma$-quanta in physical 2D space dimension.

The final result for inertial frame reads [16] (see Appendix A): 


$$
W_{I F}(\boldsymbol{r}, \theta, t) \sim \alpha^{-1} \Gamma H\left(t-\frac{\|\boldsymbol{r}\| \cos \psi}{c}\right) \exp \left[-\Gamma\left(t-\frac{\|\boldsymbol{r}\| \cos \psi}{c}\right)\right],
$$

where $\psi=\varphi-\theta, \alpha_{1} \leq \varphi \leq \alpha_{2}$ and $\alpha_{1} \leq \theta \leq \alpha_{2}$, (see Appendix C, Figure 2).

It follows from Equation (21) that under condition $\cos \psi \simeq 1$, with a probability $P \simeq 1$ the following constraint holds

$$
c t-\|\boldsymbol{r}\| \simeq 0 \text {. }
$$

The final result for non inertial frame reads [16] (see Appendix D, Figure 3):

$$
\begin{aligned}
& w_{R F}\left(r, \varphi-\omega t_{R F}, \theta, t_{R F}\right) \\
& \sim \alpha^{-1} \gamma_{r} \Gamma H\left(t_{R F}-\frac{r \cos \left(\psi-\omega t_{R F} \gamma_{r}\right)}{c_{\|r\|}}\right) \exp \left[-\Gamma \gamma_{r}\left(t_{R F}-\frac{r \cos \left(\psi-\omega t_{R F} \gamma_{r}\right)}{c_{\|r\|}}\right)\right], \\
& \text { where } c_{\mid r \|}=c \gamma_{r},\|\boldsymbol{r}\|=r, \gamma_{r}=\left[1-\frac{1}{6}\left(\frac{\omega r}{c}\right)^{2}\right] .
\end{aligned}
$$

It follows from Equation (21) that under condition $\cos \psi \simeq 1$, with a probability $P \simeq 1$ the following constraint holds

$$
c_{\|r\|} t_{R F}-r \simeq 0 \text {. }
$$

Remark 10. Note that the constraints (22) and (24) were obtained without any references to notion of the classical trajectories of $\gamma$-quanta [16]. By using proposed approach, the fundamental C. Corda result [17] can be recovered successfully by obvious way without any reference to unphysical notion of the classical trajectories of $\gamma$-quanta.

Remark 11. (i) Note that in canonical literature (see for example [4] [18] [19] [20] [21]) only unphysical specific forms of a one-photon state in one space dimension are considered. However such specific forms can be considered only as an simplification but rigorously, neither of these approximation is ever correct.This sometimes leads to misleading of the people and A. L. Kholmetskii et al. such of them.In contrast to the approach taken in Refs. [4] [18] [19] [20] we applayd a more realistic $2 \mathrm{D}$ picture [16].

(ii) Note that in paper [1] L. Kholmetskii et al. mistakenly argued that: “ The constraint (8a) used in Ref. [2] implies that the resonant $\gamma$-quanta will propagate along the radial coordinate $r$ of the rotating system, and hence, a laboratory observer would see the propagation of such $\gamma$-quanta along a curved path."

(iii) This statement from L.Kholmetskii et al. [1] wrong and based on misconception meaning mentioned above, since such $\gamma$-quanta is well localized in $k$ space and therefore is not well localized in $x$ space except unphysical 1D space dimension. Thus a laboratory observer would see nothing since there is no any curved classical path mentioned in their paper [1].

\section{Proper Time along the Interval-Valued Path. Corda's Desynchronization Term}

Let $\breve{M}^{1,3}$ be point free Minkowskian space-time endrowed with the following 
interval-valued line element in interval-valued polar coordinates

$$
\mathrm{d}[s]^{2}=c_{[\psi]}^{2} \mathrm{~d}[t]^{2}-\mathrm{d}[r]^{2}-[r]^{2} \mathrm{~d}[\varphi]^{2}-\mathrm{d}[z]^{2},
$$

where $\psi=\varphi-\theta$, see Appendix D. Note that the interval-valued line element (25) corresponding to photodetection signal which propagate with a probability $\simeq 1$ in accordance with the interval-valued law is given by Equation (C.9)-Equation (C.10), see Appendix C.

The interval-valued transformation to a non inertial frame of reference $\left(\left[t^{\prime}\right],\left[r^{\prime}\right],\left[\varphi^{\prime}\right],\left[z^{\prime}\right]\right)$ rotating at the uniform angular rate $\omega$ with respect to the starting inertial frame (26) is given by

$$
[t]=\left[t^{\prime}\right],[r]=\left[r^{\prime}\right],\left[\varphi^{\prime}\right]=[\varphi]-\omega[t] .
$$

Under transformation (26) the Equation (26) becomes the interval-valued Langevin metric in the rotating frame [16] (see Appendix D) becomes the following interval-valued line element (interval-valued Langevin metric) in rotating frame reads

$$
\begin{aligned}
& \mathrm{d}[s]^{2}=\left(1-\frac{\left[r^{\prime}\right]^{2} \omega^{2}}{c_{[\psi]-\omega t^{\prime}}^{2}}\right)\left(c_{[\psi]-\omega t^{\prime}}^{2}\right) \mathrm{d}\left[t^{\prime}\right]^{2}-2 \omega\left[r^{\prime}\right]^{2} \mathrm{~d}\left[\varphi^{\prime}\right] \mathrm{d}\left[t^{\prime}\right] \\
& -\mathrm{d}\left[r^{\prime}\right]^{2}-\left[r^{\prime}\right]^{2} \mathrm{~d}\left[\varphi^{\prime}\right]^{2}-\mathrm{d}[z]^{2} .
\end{aligned}
$$

For simplicity but without loss of generality, we consider now the following 2-dimensional interval-valued Langevin metric, see Appendix D.

$$
\begin{aligned}
\mathrm{d}[s]^{2}= & \left(1-\frac{\left[r^{\prime}\right]^{2} \omega^{2}}{c_{[\psi]-\omega t^{\prime}}^{2}}\right)\left(c_{[\psi]-\omega t}^{2}\right) \mathrm{d}\left[t^{\prime}\right]^{2}-2 \omega\left[r^{\prime}\right]^{2} \mathrm{~d}\left[\varphi^{\prime}\right] \mathrm{d}\left[t^{\prime}\right] \\
& -\mathrm{d}\left[r^{\prime}\right]^{2}-\left[r^{\prime}\right]^{2} \mathrm{~d}\left[\varphi^{\prime}\right]^{2},
\end{aligned}
$$

By substituting $\left[\varphi_{*}\right]:=\left[\underline{\varphi}_{*}, \bar{\varphi}_{*}\right]=\left[\alpha_{1}^{*}, \alpha_{2}^{*}\right]=$ const and $\left[\psi_{*}\right]=\left[\psi_{*}, \bar{\psi}_{*}\right]=\left[0, \alpha^{*}\right]$ (see Remark C.3) into Equation (28) we get

$$
\mathrm{d}[s]^{2}=\left(1-\frac{\left[r^{\prime}\right]^{2} \omega^{2}}{c_{\left[\psi_{*}\right]-\omega t^{\prime}}^{2}}\right)\left(c_{\left[\psi_{*}\right]-\omega t^{\prime}}^{2}\right) \mathrm{d}\left[t^{\prime}\right]^{2}-\mathrm{d}\left[r^{\prime}\right]^{2}=0 .
$$

The conservation law

$$
\left|\left[g_{00}\right]\right|^{1 / 2} \boldsymbol{E}_{l o c}=\text { constant }
$$

valid for any time-independent interval-valued metric with $\left[g_{0 j}\right]=0$ and for particles with both zero and non-zero rest mass. It describes how the locally measured energy of any particle or photon changes (is "red-shifted" or "blueshifted") as it climbs out of or falls into a static gravitational field. For a particle of zero rest mass as photon, the locally measured energy $E_{l o c}$, and wavelength $\lambda_{\text {loc }}$, are related by $\boldsymbol{E}_{\text {loc }}=\hbar / \lambda_{\text {loc }}=\hbar v_{\text {loc }}$, where $\hbar$ is Planck's constant. Consequently, the law of energy shift can be rewritten as

$$
\|\left.\left[g_{00}\right]\right|^{-1 / 2} \lambda_{\text {loc }}=\text { constant. }
$$

Therefore, from Equation (31), one gets 


$$
\frac{\left[\Delta \tau_{10}\right]-\left[\Delta \tau_{11}\right]}{\tau_{1}}=1-\frac{1}{\sqrt{1-\frac{R^{2} \omega^{2}}{\left.c_{\left[\psi_{*}\right]}^{2}\right]-\omega t^{\prime}}}} \simeq 1-\frac{1}{\sqrt{1-\frac{v^{2}}{c^{2}}}} \simeq-\frac{1}{2} \frac{v^{2}}{c^{2}},
$$

where we use the proper time $\tau$ rather than the wavelength $\lambda$ and where $\left[\Delta \tau_{10}\right]$ is the delay of the emitted radiation, $\left[\Delta \tau_{11}\right]$ is the delay of the received radiation, $\tau_{1} \simeq R c_{[\psi]}^{-1}, R$ is the radial coordinate of the absorber (see Figure $2 \&$ Figure 3) and $v=R \omega$, where $\omega$ is the tangential velocity of the absorber. In a gravitational field, the rate $\mathrm{d}[\tau]$ of the proper time is related to the rate $\left[\mathrm{d} t^{\prime}\right]$ of the coordinate time by

$$
\mathrm{d}[\tau]=\sqrt{1-\frac{r^{\prime 2} \omega^{2}}{c_{\left[\psi_{*}\right]-\omega\left[t^{\prime}\right]}^{2}}}\left[\mathrm{~d} t^{\prime}\right] .
$$

Using now again Equation (26), we get

$$
c_{[\psi]}^{2}\left[\mathrm{~d} t^{\prime}\right]^{2}=c_{[\psi]}^{2}[\mathrm{~d} t]^{2}=\mathrm{d}[r]^{2}=\mathrm{d}\left[r^{\prime}\right]^{2},
$$

where the equality

$$
c_{[\psi]}^{2}[\mathrm{~d} t]^{2}=\mathrm{d}[r]^{2}
$$

follows from the issue that in the laboratory frame photodetection signal propagate with a probability $\simeq 1$, in accordance with the following interval-valued law

$$
[r] \simeq c_{[\psi]}[t],[\varphi]=\left[\alpha_{2}, \alpha_{1}\right] \text {, }
$$

see Appendix C, Equation (C.9). Hence, Equation (33) becomes

$$
c_{[\psi]}^{2} \mathrm{~d}[\tau]=\sqrt{1-\frac{r^{\prime 2} \omega^{2}}{c_{\left[\psi_{*}\right]-\omega\left[t^{\prime}\right]}^{2}}}\left[\mathrm{~d} r^{\prime}\right] .
$$

Note that the Equation (15) is well approximated by

$$
c_{[\psi]}^{2} \mathrm{~d}[\tau] \simeq\left(1-\frac{1}{2} \frac{\left[r^{\prime}\right]^{2} \omega^{2}}{c_{[\psi *}^{2}}+\cdots\right)\left[\mathrm{d} r^{\prime}\right] .
$$

Therefore, the second contribution of order $v^{2} / c_{[\psi]}^{2}$ to the variation of proper time reads

$$
c_{[\psi]}\left[\Delta \tau_{2}\right] \simeq \int_{0}^{\left[r_{1}^{\prime}\right]}\left(1-\frac{1}{2} \frac{\left[r^{\prime}\right]^{2} \omega^{2}}{c_{\left[\psi_{*}\right]}^{2}}+\cdots\right)\left[\mathrm{d} r^{\prime}\right]-\left[r_{1}^{\prime}\right]=-\frac{1}{6}\left[r_{1}^{\prime}\right]^{2} \frac{v^{2}}{c_{[\psi]}^{2}} .
$$

Note that $\left[r^{\prime}\right] \simeq c_{[\psi]}\left[\tau_{1}\right]$ is the radial distance between the source and the detector. Then, one gets the Corda's desynchronization term

$$
z_{2}=\frac{\left[\Delta \tau_{2}\right]}{\left[\tau_{1}\right]}=-\frac{1}{6} \frac{v^{2}}{c_{[\psi]}^{2}} .
$$

Under conditions of Kündig experiment [9] (40) is well approximated by

$$
z_{2} \simeq-\frac{1}{6} \frac{v^{2}}{c^{2}}
$$




\section{Conclusions}

By using proposed approach based on point-free Lorentzian geometry [16], the fundamental C. Corda result [17] [22] [23] [24] recovered successfully by obvious way without any reference to unphysical notion of the classical trajectories of $\gamma$-quanta.

In additional note that YARK group papers [30] [31] [32] wrong and must be rejected since in contrast with Kündig [9] YARK group did not measure the sign of the energy shift between emission and absorption lines but attributed this sign by own ubnormal meaning based on wrong Equation (1).

\section{Conflicts of Interest}

The author declares no conflicts of interest regarding the publication of this paper.

\section{References}

[1] Kholmetskii, A.L., Yarman, T., Yarman, O. and Arik, M. (2020) Analyses of Mossbauer Experiments in a Rotating System: Proper and Improper Approaches. Annals of Physics, 418, Article ID: 168191. https://doi.org/10.1016/j.aop.2020.168191

[2] Podosenov, S.A., Foukzon, J. and Men'kova, E.R. (2020) Comment on “The Mossbauer Rotor Experiment and the General Theory of Relativity" [Ann. Physics 368 (2016) 258-266]. Annals of Physics, 413, Article ID: 168047. https://doi.org/10.1016/j.aop.2019.168047

[3] Tonnela, M.A. (1966) The Principles of Electromagnetic Theory and of Relativity.

[4] Gilbert, G., Aspect, A. and Fabre, C. (2010) Introduction to Quantum Optics: From the Semi-Classical Approach to Quantized Light.

[5] Hay, H.J., et al. (1960) Measurement of the Red Shift in an Accelerated System Using the Mössbauer Effect in $\mathrm{Fe}^{57}$. Physical Review Letters, 4, 165. https://doi.org/10.1103/PhysRevLett.4.165

[6] Champeney, D.C. and Moon, P.B. (1961) Absence of Doppler Shift for Gamma Ray Source and Detector on Same Circular Orbit. Proceedings of the Physical Society, 77, 350. https://doi.org/10.1088/0370-1328/77/2/318

[7] Hay, H.J. (1960) Measurement of the Red Shift in an Accelerated System Using the Mössbauer Effect in $\mathrm{Fe}^{57}$. Physical Review Letters, 4, 165.

[8] Granshaw, T.E. and Hay, H.J. (1963) International School of Physics Enrico Fermi. Academic Press, New York, 220.

[9] Kündig, W. (1963) Measurement of the Transverse Doppler Effect in an Accelerated System. Physical Review, 129, 2371. https://doi.org/10.1103/PhysRev.129.2371

[10] Møller, C. (1973) The Theory of Relativity. Clarendon Press, Oxford.

[11] Okun, L.B., Selivanov, K.G. and Telegdi, V.L. (2000) On the Interpretation of the Redshift in a Static Gravitational Field. American Journal of Physics, 68, 115. https://doi.org/10.1119/1.19382

[12] Yarman, T., Kholmetskii, A.L., Akkuş, B., Öktem, Y., Susam, L.A. and Missevitch, O.V. (2016) Novel Mössbauer Experiment in a Rotating System and the Extra-Energy Shift between Emission and Absorption Lines. Canadian Journal of Physics, 94, 780-789. https://doi.org/10.1139/cjp-2015-0063 
[13] Mashhoon, B. (1990) Limitations of Spacetime Measurements. Physics Letters A, 143, 176-182. https://doi.org/10.1016/0375-9601(90)90734-6

[14] Mashhoon, B. (1990) The Hypothesis of Locality in Relativistic Physics. Physics Letters A, 145, 147-153. https://doi.org/10.1016/0375-9601(90)90670-J

[15] Mashhoon, B. and Muench, U. (2002) Length Measurement in Accelerated Systems. Annalen der Physik, 11, 532-547. https://doi.org/10.1002/1521-3889(200208)11:7<532::AID-ANDP532>3.0.CO;2-3

[16] Podosenov, S.A., Foukzon, J. and Men'kova, E.R. (2020) Erratum to Comment on "The Mössbauer Rotor Experiment and the General Theory of Relativity" [Ann. Physics 368 (2016) 258-266]. Annals of Physics C, 423, Article ID: 168329. https://doi.org/10.1016/j.aop.2020.168329

[17] Iovane, G. and Benedetto, E. (2019) Coordinate Velocity and Desynchronization of Clocks. Annals of Physics (New York), 403, 106-111. https://doi.org/10.1016/j.aop.2019.02.003

[18] Chiao, R. and Garrison, J. (2014) Quantum Optics (Oxford Graduate Texts). (Inglés) Reprint Edition, 736 p.

[19] Milonni, P.W. (2019) An Introduction to Quantum Optics and Quantum Fluctuations (Oxford Graduate Texts). (Inglés) Tapa dura-Ilustrado.

[20] Chan, K.W., Law, C.K. and Localized, J.H. (2002) Single-Photon Wave Functions in Free Space. Physical Review Letters, 88, Article ID: 100402.

https://doi.org/10.1103/PhysRevLett.88.100402

[21] Fedorov, M.V., Efremov, M.A., Kazakov, A.E., Chan, K.W., Law, C.K. and Eberly, J.H. (2005) Spontaneous Emission of a Photon: Wave-Packet Structures and Atom-Photon Entanglement. Physical Review A, 72, Article ID: 032110. https://doi.org/10.1103/PhysRevA.72.032110

[22] Corda, C. (2015) Interpretation of Mössbauer Experiment in a Rotating System: A New Proof for General Relativity. Annals of Physics, 355, 360-366. https://doi.org/10.1016/j.aop.2015.02.021

[23] Corda, C. (2018) New Proof of General Relativity through the Correct Physical Interpretation of the Mossbauer Rotor Experiment. International Journal of Modern Physics D, 27, Article ID: 1847016. https://doi.org/10.1142/S0218271818470168

[24] Corda, C. (2019) Mossbauer Rotor Experiment as New Proof of General Relativity: Rigorous Computation of the Additional Effect of Clock Synchronization. International Journal of Modern Physics D, 28, Article ID: 1950131. https://doi.org/10.1142/S0218271819501311

[25] Concilio, A.D. (2013) Point-Free Geometries: Proximities and Quasi-Metrics. Mathematics in Computer Science, 7, 31. https://doi.org/10.1007/s11786-013-0140-2

[26] Tozzi, A. and Peters, J.F. (2019) Points and Lines inside Human Brains. Cognitive Neurodynamics, 13, 417-428. https://doi.org/10.1007/s11571-019-09539-8

[27] Alefeld, G. and Mayer, G. (2000) Interval Analysis: Theory and Applications. Journal of Computational and Applied Mathematics, 121, 421-464. https://doi.org/10.1016/S0377-0427(00)00342-3

[28] Moore, R.E., Kearfott, R.B. and Cloud, M.J. (2009) Introduction to Interval Analysis. 213.

[29] Landau, L.D. and Lifshitz, E.M. (1971) The Classical Theory of Fields. Vol. 2 Course of Theoretical Physics. 4th Edition, Butterworth-Heinemann, Oxford.

[30] Kholmetskii, A.L., Yarman, T., Missevitch, O.V. and Rogozev, B.I. (2009) A Mössbauer Experiment in a Rotating System on the Second-Order Doppler Shift: 
Confirmation of the Corrected Result by Kündig. Physica Scripta, 79, Article ID: 065007. https://doi.org/10.1088/0031-8949/79/06/065007

[31] Kholmetskii, A.L., Yarman, T. and Missevitch, O.V. (2011) Mossbauer Experiment in a Rotating System on the Time Dilation Effect. International Journal of Physical Sciences, 6, 84-92.

[32] Kholmetskii, A.L., Yarman, T., Arik, M. and Missevitch, O.V. (2015) Novel Mössbauer Experiment in a Rotating System: Extra Energy Shift Confirmed. AIP Conference Proceedings, 1648, Article ID: 510011.

https://doi.org/10.1063/1.4912716 


\section{Appendix. A. Two-Dimensional Wave Packet and Corresponding Conditional Photodetection Probability Density Function}

Consider a one-photon state of the form

$$
|1\rangle=\sum_{l} c_{l}(\varphi)\left|0, \cdots, n_{l}=1,0, \cdots\right\rangle=\sum_{l} c_{l}(\varphi)\left|1_{l}\right\rangle,
$$

where

$$
\sum_{l}\left|c_{l}(\varphi)\right|^{2}=\alpha^{-1}
$$

$\alpha_{1} \leq \varphi \leq \alpha_{2}, \alpha=\alpha_{1}-\alpha_{2}$, see Figure 1 . Thus normalizability condition reads

$$
\int_{\alpha_{1}}^{\alpha_{1}} \mathrm{~d} \varphi \sum_{l}\left|c_{l}(\varphi)\right|^{2}=1
$$

A state (A.1) changes in time and the state is given at time $t$ by

$$
|1(t)\rangle=\sum_{l} c_{l}(\varphi) \exp \left(-i \omega_{l} \times t\right)\left|1_{l}\right\rangle .
$$

The photodetection signal at time $t$ and fixed point $\boldsymbol{r}=(r, \varphi), r=\|\boldsymbol{r}\|$ (see Figure 2) is given by [2]:

$$
w(\boldsymbol{r}, t)=w(\|\boldsymbol{r}\|, \varphi, t)=s \| \hat{\boldsymbol{E}}^{(+)}(\boldsymbol{r})|1(t)\rangle \|^{2}=s\left|E^{(+)}(\boldsymbol{r}, t)\right|^{2},
$$

where $\varphi$ is angle $(\boldsymbol{r}, \boldsymbol{X})$ between axis $\boldsymbol{X}$ and vector $\boldsymbol{r}$ (see Figure 1), $s$ is the sensitivity of detector and where

$$
\begin{gathered}
E^{(+)}(\boldsymbol{r}, t)=\sum_{l} c_{l}\left(\boldsymbol{k}_{l}, \varphi, \theta\right) \boldsymbol{\varepsilon}_{l} \exp \left[i\left(\boldsymbol{k}_{l} \cdot \boldsymbol{r}-\omega_{l} \times t\right)\right] \\
c_{l}\left(\boldsymbol{k}_{l}, \varphi, \theta\right)=\Delta(\varphi, \theta) c_{l}\left(\boldsymbol{k}_{l}\right) \text { and } \\
\boldsymbol{k}_{l} \cdot \boldsymbol{r}=\left\|\boldsymbol{k}_{l}\right\| \times\|\boldsymbol{r}\| \times \cos \left(\boldsymbol{r}, \boldsymbol{k}_{l}\right)=\omega_{l} \times\|\boldsymbol{r}\| \times \cos (\varphi, \theta),
\end{gathered}
$$

see Figure 2.

Remark A.1. (i) We consider now a set of coefficients $c_{l}=c_{l}\left(\boldsymbol{k}_{l}, \varphi, \theta\right)$ different from zero for values of $\boldsymbol{k}_{l}$ distributed over some bounded region $G_{\boldsymbol{k}_{0}}$ of $\boldsymbol{k}$-space of extent $\delta k_{x}, \delta k_{y}, \delta k_{z}$ about a value $\boldsymbol{k}_{0}$ and in addition are different from zero for values of $\varphi$ and values of $\theta$ such that $\alpha_{1} \leq \varphi \leq \alpha_{2}$ and $\alpha_{1} \leq \theta \leq \alpha_{2}$, see Figure 2. Thus

$$
\Delta(\varphi, \theta)=1 \text { if } \varphi, \theta \in\left[\alpha_{1}, \alpha_{2}\right]
$$

and

$$
\Delta(\varphi, \theta) \equiv 0 \text { if } \varphi \notin\left[\alpha_{1}, \alpha_{2}\right] \text { or } \theta \notin\left[\alpha_{1}, \alpha_{2}\right]
$$

(ii) We thus obtain, at time $t=0$, a $2 \mathrm{D}$ wave packet localized in a volume of $\boldsymbol{x}$-space with dimensions of the order of $\hbar\left(\delta k_{x}\right)^{-1}, \hbar\left(\delta k_{y}\right)^{-1}$. When the same set of coefficients $c_{l}=c_{l}\left(\boldsymbol{k}_{l}, \varphi\right)$ is substituted into (A.1), we thus obtain a photodetection signal (A.4) that differs from zero only within some bounded region $G_{x}$ of $\boldsymbol{x}$-space of extent $\hbar\left(\delta k_{x}\right)^{-1}, \hbar\left(\delta k_{y}\right)^{-1}$.

(iii) The volume of this region generally increases without limit as time goes by, and this in each space dimension, there are specific form of a one-photon 
state in 1D space dimension for which the spreading effect does not occur [3]. This kind of wave packet is not physically realistic, in the sense that it extends infinitely in the plane perpendicular to $\boldsymbol{n}$.

(iv) However, there are specific forms for which the spreading effect does not occur dramatically in 2D and 3D space dimension. An nontrivial example is the 2D wave packet we are about to discuss. Consider now the case in which the wavevectors $\boldsymbol{k}_{l}$ associated with the non-zero coefficients $c_{l}$ are all parallel to the same unit vector $\boldsymbol{n}$ (see Figure 2), i.e.

$$
\boldsymbol{k}_{l}=\frac{\omega_{l}}{C} \boldsymbol{n}=\frac{2 \pi l}{L} \boldsymbol{n} .
$$

where $L$ is an arbitrary quantization length. The function $E^{(+)}(\boldsymbol{r}, t)$ given by Equation (A.6) then takes the form

$$
E^{(+)}(\boldsymbol{r}, t)=\Delta(\varphi, \theta) \sum_{l} c_{l}\left(\omega_{l}\right) E_{l}^{(1)} \boldsymbol{\varepsilon}_{l} \exp \left[i \omega_{l}\left(\frac{\boldsymbol{r} \cdot \boldsymbol{n}}{c}-t\right)\right] .
$$

Thus the photodetection probability (A.4) depends on space and time only through the quantity

$$
\tau_{\psi}=t-\boldsymbol{r} \cdot \boldsymbol{n} / c=t-\frac{\|\boldsymbol{r}\| \cos (\boldsymbol{r}, \boldsymbol{n})}{c}=t-\frac{\|\boldsymbol{r}\| \cos \psi}{c},
$$

where $\psi=\varphi-\theta$, (see Figure 2). Let us consider the case where all the modes have the same polarization $\varepsilon$. The coefficients $c$ then depend only on the frequency $\omega$, and a wave packet can be formed by considering a distribution peaking at some $\omega_{0}$, described by

$$
c_{l}\left(\omega_{l}\right)=g\left(\omega_{l}-\omega_{0}\right),
$$

where $g(\xi), \xi=\omega_{1}-\omega_{0}$ is a function centered on 0 and having a typical half-width $\delta \omega$ that is small compared with $\omega_{0}$. The function (A.11) will then be proportional to the Fourier transform $\hat{g}\left(\tau_{\psi}\right)$ of $g(\xi)$, yielding a wave packet with width of the order of $1 / \delta \omega$. To carry out the calculation explicitly, the sum in (A.11) is replaced by an integral, introducing the one-dimensional mode density deduced from (A.10):

$$
\frac{\mathrm{d} l}{\mathrm{~d} \omega_{l}}=\frac{L}{2 \pi c} .
$$

The final result reads

$$
\begin{aligned}
E^{(+)}(\boldsymbol{r}, t) & \sim \boldsymbol{\varepsilon} \sqrt{\frac{\hbar \omega_{0}}{2 \varepsilon_{0} L}} \frac{L \Delta(\varphi, \theta)}{2 \pi c} \int_{-\infty}^{+\infty} \mathrm{d} \omega_{l} c\left(\omega_{l}\right) \exp \left(-i \omega_{l} \tau_{\psi}\right) \\
& =\frac{\boldsymbol{\varepsilon} \Delta(\varphi, \theta)}{2 \pi c} \sqrt{\frac{\hbar \omega_{0} L}{2 \varepsilon_{0}}} \exp \left[-i \omega_{0}\left(t-\frac{\|\boldsymbol{r}\| \cos \psi}{c}\right)\right],
\end{aligned}
$$

where

$$
\hat{g}\left(\tau_{\psi}\right)=\frac{1}{\sqrt{2 \pi}} \int_{-\infty}^{+\infty} \mathrm{d} \xi g(\xi) \exp \left(-i \xi \tau_{\psi}\right) .
$$

The photodetection probability density (A.5) reads 


$$
w(\boldsymbol{r}, t) \sim \alpha^{-1} \Delta(\varphi, \theta)\left|\hat{g}\left(t-\frac{\|\boldsymbol{r}\| \cos (\boldsymbol{r}, \boldsymbol{n})}{c}\right)\right|^{2} .
$$

Spontaneous emission by a single atom in an excited state gives a one-photon wave packet. For this case one obtains the coefficients

$$
c_{l}\left(\omega_{l}\right)=\frac{K}{\omega_{l}-\omega_{0}+i \Gamma / 2} .
$$

Note that the emitted light spectrum is described by a Lorentzian line centred at $\omega_{0}$, with width $\Gamma$ at half-maximum:

$$
\left|c_{l}\left(\omega_{l}\right)\right|^{2}=\frac{K}{\left(\omega_{l}-\omega_{0}\right)^{2}+\Gamma^{2} / 4} .
$$

We now write $E^{(+)}(\boldsymbol{r}, t)$ in the form (A.15). The Fourier transform of

$$
g(\xi)=\frac{K}{\xi+i \Gamma / 2} .
$$

one obtains

$$
\hat{g}\left(\tau_{\psi}\right)=K \sqrt{2 \pi} H\left(\tau_{\psi}\right) \exp \left(-\frac{\Gamma}{2} \tau_{\psi}\right)
$$

where $H\left(\tau_{\psi}\right)$ is the Heaviside step function, equal to 0 for $\tau<0$ and 1 for $\tau \geq 0$.

The final result reads

$$
w(\boldsymbol{r}, \theta, t) \sim \alpha^{-1} \Gamma H\left(t-\frac{\|\boldsymbol{r}\| \cos \psi}{c}\right) \exp \left[-\Gamma\left(t-\frac{\|\boldsymbol{r}\| \cos \psi}{c}\right)\right],
$$

where $\psi=\varphi-\theta$, (see Figure 2).

Remark A.2. (i) Note that the probability density $w(t, r)$ cannot be considered as the wave function of the photon, whose squared modulus, suitably normalized, gives the probability density for the presence of the photon, measured by a photodetector (see [3], 5.6.).

(ii) It should not be thought that there is a position operator $\hat{\boldsymbol{r}}$ for the photon corresponding to measurements by a photodetector. Therefore the probability density $w(t, \boldsymbol{r})$ cannot be considered as the probability density of finding a photon exactly at point $r \in \mathbb{R}^{2}$, but rather as an average probability density over some small area which cannot be smaller than $\lambda_{\min }^{2}$, where $\lambda_{\min }$ is the wavelength associated with the maximum frequency to which the detector is sensitive.

(iii) Thus the quantity $w(t, r) \mathrm{d} t \delta \theta$ is the probability of detecting a click near point $\boldsymbol{r}=(r, \theta)$, between $t$ and $t+\mathrm{d} t$, i.e. by a detector with angular size $\delta \theta \ll 1$, where $\lambda_{\min } \leq r \delta \theta$ and located at the point $r \in \mathbb{R}^{2}$.

Remark A.3. Note that normalizing factor in Equation (A.22) contains a multiplier $\alpha^{-1}$ with $\alpha=\alpha_{1}-\alpha_{2} \quad \psi \ll 2 \pi$ rad and thus $\cos \psi \simeq 1$. Therefore the conditions is given by Equation (B.5) and by Equation (B.6) [see Apendix B] are satisfied, since 


$$
\int_{\underline{t}}^{\bar{t}} \int_{[0,2 \pi]\left[\alpha_{2}, \alpha_{1}\right]} w(r, \varphi, \theta, t) \mathrm{d} t \mathrm{~d} \varphi=0,
$$

and

$$
P\left([t],[r],[\varphi],\left[\theta^{\prime}\right]\right) \simeq \int_{\underline{t}}^{t} \int_{\alpha_{2}^{\prime}}^{\alpha_{1}^{\prime}} w(r, \varphi, \theta, t) \mathrm{d} t \mathrm{~d} \varphi<1 / 2,
$$

where $[t]=[\underline{t}, \bar{t}],\left[\theta^{\prime}\right]=\left[\alpha_{2}^{\prime}, \alpha_{1}^{\prime}\right] \varsubsetneqq 0.5\left[\alpha_{2}, \alpha_{1}\right]$.

\section{Appendix. B. Quantum Measurement on Inertial Relativistic Frame of Reference. Point-Free Minkowski Geometry}

In this appendix we introduce point-free Minkowski geometry [16] [25] [26], related to relativistic quantum measurement on inertial relativistic frame of reference.

If we are to suppose that a quantum particle at a definite position $x=\left(x_{1}, x_{2}, x_{3}\right) \in \mathbb{R}^{3}$ at instant $t \in[0, T]$ is to be assigned a state vector $|t, x\rangle$, and if further we are to suppose that the possible positions $x_{i}, i=1,2,3$ are continuous over the range $(-\infty,+\infty)$ and that the associated states are complete, then we are lead to requiring that any state $\left|\psi_{t}\right\rangle$ of the particle at instant $t \in[0, T]$ must be expressible as

$$
\left|\psi_{t}\right\rangle=\int_{-\infty}^{\infty} \mathrm{d}^{3} x|t, x\rangle\left\langle x, t \mid \psi_{t}\right\rangle
$$

with the states $|t, x\rangle$ by $\delta$ function normalised, i.e. $\left\langle x, t \mid t^{\prime}, x^{\prime}\right\rangle=\delta^{3}\left(x-x^{\prime}\right) \delta\left(t-t^{\prime}\right)$. However well known that the notion of preparing a particle in a state $|t, x\rangle$ does not even make any physical sense. The resolution of this impasse involves recognizing that the measurement of the position of a particle is, in practice, only ever done to within the accuracy, $\delta x=\left(\delta x_{1}, \delta x_{2}, \delta x_{3}\right)$ say, of the measuring apparatus. In other words, rather than measuring the precise position of a particle, what is measured is its position as lying somewhere in a range $\left(x_{i}-\frac{1}{2} \delta x_{i}, x_{i}+\frac{1}{2} \delta x_{i}\right), i=1,2,3$ Therefore if the particle is in some state $\left|\psi_{t}\right\rangle$, we can recognize that the probability $P_{\left|\psi_{t}\right\rangle}(t, x, \delta t, \delta x)$ of getting a result $x$ with an accuracy of $\delta x$ between instants $t-\delta t$ and $t+\delta t$. will be given by

$$
P_{\left|\psi_{t}\right\rangle}(t, x, \delta t, \delta x)=\int_{t-\delta t}^{t+\delta t} \mathrm{~d} t^{\prime} \int_{x_{1}-\frac{1}{2} \delta x_{1}}^{x_{1}+\frac{1}{2} \delta x_{2}-\frac{1}{2} \delta x_{1}} \int_{x_{2}+\frac{1}{2} \delta x_{2}-\frac{1}{2} \delta x_{3}}^{x_{3}+\frac{1}{2} \delta x_{3}} \mathrm{~d}^{3} x^{\prime}\left|\left\langle x^{\prime}, t^{\prime} \mid \psi\right\rangle\right|^{2} .
$$

1. We assume now that at point $x \in \mathbb{R}^{3}$ the following estimate is satisfied

$$
P_{\left|\psi_{t}\right\rangle}(t, x, \delta t, \delta x)=1-c_{1} \exp \left(-c_{2}\|\delta x\|\right)-c_{3} \exp \left(-c_{4} \delta t\right) \simeq 1,
$$

where $c_{1}, c_{3} \leq 1, c_{2}, c_{4} \gg 1$ are positive constants suth that $c_{2}\|\delta x\| \gg 1, c_{4} \delta t \gg 1$ and $\|\delta x\| \ll 1, \delta t \ll 1,\|\delta x\|=\sqrt{\left(\delta x_{1}\right)^{2}+\left(\delta x_{2}\right)^{2}+\left(\delta x_{3}\right)^{2}}$.

Remark B.1. Note that only under condition (B.3) the notion of position of a quantum particle at instant $t$ holds in well approximation relevant to classical sense, i.e. as a definite point $x \in \mathbb{R}^{3}$. 
2. We assume now that there exists continuous vector-function $x_{t}:[0, T] \rightarrow \mathbb{R}^{3}$ such that for all $t \in[0, T]$ the following estimate is satisfied

$$
P_{\left|\psi_{t}\right\rangle}(t, x, \delta t, \delta x)=1-c_{1} \exp \left(-c_{2}\|\delta x\|\right)-c_{3} \exp \left(-c_{4} \delta t\right) \simeq 1,
$$

where $c_{1}, c_{3} \leq 1, c_{2}, c_{4} \gg 1$ are positive constants suth that $c_{2}\|\delta x\| \gg 1, c_{4} \delta t \gg 1$ and $\|\delta x\| \ll 1, \delta t \ll 1$.

Remark B.2. Note that only under condition (B.4) the notion of trajectory of a quantum particle holds in well approximation relevant to classical sense, i.e. as continuous vector-function $x_{t}:[0, T] \rightarrow \mathbb{R}^{3}$.

3. We assume now that at point $x \in \mathbb{R}^{3}$ the following estimates are satisfied

$$
\begin{aligned}
& P_{\left|\psi_{t}\right\rangle}(t, x, \delta t, \delta x)=1-c_{1} \exp \left(-c_{2}\|\delta x\|\right) \simeq 1, \\
& P_{\left|\psi_{t}\right\rangle}\left(t, x, \frac{\delta t}{2}, \frac{\delta x}{2}\right)<1 / 2
\end{aligned}
$$

where $c_{1}, c_{1}$ are positive constants and $\|\delta x\|>1, \delta t>1$ and

$$
P_{\left|\psi_{t}\right\rangle}\left(\mathbb{R}^{3} \backslash \Delta \mid t, x, \delta t, \delta x\right)=c_{3} \exp \left(-c_{4}\|\delta x\|\right) \approx 0,
$$

where $c_{4} \gg c_{2}$, and

$$
P_{\left|\psi_{t}\right\rangle}\left(\mathbb{R}^{3} \backslash \Delta \mid t, x, \delta t, \delta x\right)=\int_{t-\delta t}^{t+\delta t} \mathrm{~d} \tau \int_{\mathbb{R}^{3} \backslash \Delta} \mathrm{d}^{3} x^{\prime}\left|\left\langle x^{\prime} \mid \psi_{\tau}\right\rangle\right|^{2} .
$$

where $\Delta=\Delta_{1} \times \Delta_{2} \times \Delta_{3}, \Delta_{i}=0.5\left[x_{i}-\frac{1}{2} \delta x_{i}, x_{i}+\frac{1}{2} \delta x_{i}\right], i=1,2,3$.

Remark B.3. Note that under conditions (B.5)-(B.7) the notion of position of a quantum particle at instant $t$ no longer holds in well approximation relevant to classical sense and well defined only by using notion of the interval numbers [16].

4. We assume now that there exists continuous vector-function $x_{t}:[0, T] \rightarrow \mathbb{R}^{3}$ such that for all $t \in[0, T]$ the following estimates are satisfied

$$
\begin{aligned}
& P_{\left|\psi_{t}\right\rangle}\left(t, x_{t}, \delta t, \delta x_{t}\right)=1-c_{1} \exp \left(-c_{2}\left\|\delta x_{t}\right\|\right) \simeq 1, \\
& P_{\left|\psi_{t}\right\rangle}\left(t, x_{t}, \frac{\delta t}{2}, \frac{\delta x_{t}}{2}\right)<1 / 2,
\end{aligned}
$$

where $c_{1}, c_{1}$ are positive constants and for all $t \in[0, T],\left\|\delta x_{t}\right\|>1, \delta t>1$, and

$$
P_{\left|\psi_{t}\right\rangle}\left(\mathbb{R}^{3} \backslash \Delta \mid t, x_{t}, \delta t, \delta x_{t}\right)=c_{3} \exp \left(-c_{4}\left\|\delta x_{t}\right\|\right) \approx 0,
$$

where $c_{4} \gg c_{2}$. and

$$
P_{\left|\psi_{t}\right\rangle}\left(\mathbb{R}^{3} \backslash \Delta \mid t, x_{t}, \delta t, \delta x_{t}\right)=\int_{t-\delta t}^{t+\delta t} \mathrm{~d} t^{\prime} \int_{\mathbb{R}^{3} \backslash \Delta} \mathrm{d}^{3} x^{\prime}\left|\left\langle x^{\prime}, t^{\prime} \mid \psi_{t^{\prime}}\right\rangle\right|^{2} .
$$

where $\Delta=\Delta_{1, t} \times \Delta_{2, t} \times \Delta_{3, t}, \Delta_{i, t}=\left[x_{i, t}-\frac{1}{2} \delta x_{i, t}, x_{i, t}+\frac{1}{2} \delta x_{i, t}\right], i=1,2,3$.

Remark B.4. Note that under conditions (B.8)-(B.10) the notion of trajectory of a quantum particle no longer holds in well approximation relevant to classical sense and well defined only by using notion of the interval-valued function [27] 
[28].

We rewrite now the Equation (B.5) using notion of the interval number in the following form

$$
P_{\left|\psi_{t}\right\rangle}([t],[x])=\int_{\underline{t}}^{\bar{t}} \mathrm{~d} t^{\prime} \int_{\underline{x_{1}}}^{\bar{x}_{1}} \int_{\underline{\underline{x}_{2}}}^{\bar{x}_{2}} \int_{\underline{x_{3}}}^{\bar{x}_{3}} \mathrm{~d}^{3} x^{\prime}\left|\left\langle x^{\prime}, t^{\prime} \mid \psi\right\rangle\right|^{2} \simeq 1,
$$

where $[t]=[\underline{t}, \bar{t}],[x]=\left(\left[x_{1}\right],\left[x_{2}\right],\left[x_{3}\right]\right) \in \mathbf{I}\left(\mathbb{R}^{3}\right)$ is interval number:

$$
\left[x_{i}\right]=[\underline{x}, \bar{x}], \underline{x}_{i}=x_{i}-\frac{1}{2} \delta x_{i}, \bar{x}_{i}=x_{i}+\frac{1}{2} \delta x_{i},
$$

$i=1,2,3$, where $[t]=[\underline{t}, \bar{t}]$

Remark B.5. Thus the result of quantum measurement of the position of a particle that is alwais interval number: $[x]$ is given by Equation (B.4) of getting a result $[x]$ with the probability $P_{\left|\psi_{t}\right\rangle}([t],[x]) \simeq 1$.

Remark B.6. Let $[a]=[\underline{a}, \bar{a}] ;[b]=[\underline{b}, \bar{b}]$ be real compact intervals. The following rules hold: (i) $[a]+[b]=[\underline{a}+\underline{b}, \bar{a}+\bar{b}]$; (ii) $[a]-[b]=[\underline{a}-\underline{b}, \bar{a}-\bar{b}]$;

(iii) $[a] \cdot[b]=[\min \{\underline{a} \underline{b}, \underline{a} \bar{b}, \bar{a} \underline{b}, \bar{a} \bar{b}\}, \max \{\underline{a} \underline{b}, \underline{a} \bar{b}, \bar{a} \underline{b}, \bar{a} \bar{b}\}]$;

(iv) $[b]^{-1}=\left\{b^{-1} \mid b \in[b]\right\}$ if $0 \notin[b]$, thus $[a] /[b] \triangleq[a] \cdot[b]^{-1}$.

If $\underline{a}=\bar{a}=a$, i.e., if $[a]$ consists only of the element $a$, then we identify the real number $a \in \mathbb{R}$ with the degenerate interval $[a, a]$ keeping the canonical notation, i.e., $a \equiv[a, a]$.

It is easy to prove that the set $\mathbf{I}(\mathbb{R})$ of real compact intervals is closed with respect to these operations.

Remark B.1. Unfortunately, $(\mathbf{I}(\mathbb{R}),+, \cdot)$ is neither a field nor a ring. The structures $(\mathbf{I}(\mathbb{R}),+)$ and $(\mathbf{I}(\mathbb{R}) /\{0\}, \cdot)$ are commutative semigroups with the neutral elements 0 and 1 , respectively, but they are not groups. A nondegenerate interval $[a]$ has no inverse with respect to addition or multiplication. Even the distributive law has to be replaced by the so-called subdistributivity:

$[a] \cdot([b]+[c]) \subseteq[a] \cdot[b]+[a] \cdot[c]$.

Remark B.2. (i) In order to avoid difficultness mentioned above we replace any interval number $[x]=[\underline{x}, \bar{x}]$ by an continuous function $\left[x_{\zeta}\right]:[0,1] \rightarrow[\underline{x}, \bar{x}]$ such that the following conditions are satisfied [16]:

$$
\underline{x}=\inf _{\varsigma \in[0,1]}\left[x_{\zeta}\right], \sup _{\zeta \in[0,1]}\left[x_{\zeta}\right]=\bar{x}
$$

where $\underline{x} \leq\left[x_{\zeta}\right] \leq \bar{x}$.

(ii) We usually keeping for short the canonical notation $[x]$, i.e., $[x] \triangleq\left[x_{\zeta}\right], \zeta \in[0,1]$

(iii) Note that the notation $[x]=[\underline{x}, \bar{x}]$ mean $\underline{x} \leq\left[x_{\zeta}\right] \leq \bar{x}$, but not real compact interval: $\{x \mid \underline{x} \leq x \leq \bar{x}\}$.

(iv) We also keeping the notation $x_{\zeta}$ which mean a value of the function $\left[x_{\zeta}\right]$ in a given point $\zeta \in[0,1]$.

Let $[a]=[\underline{a}, \bar{a}] ;[b]=[\underline{b}, \bar{b}]$. The following rules hold:

(i) $[a]+[b] \triangleq\left[a_{\zeta}\right]+\left[b_{\zeta}\right]=\left[a_{\zeta}+b_{\zeta}\right], \zeta \in[0,1]$, thus $[a]+[b]=[b]+[a]$;

(ii) $[a]-[b] \triangleq\left[a_{\zeta}\right]-\left[b_{\zeta}\right]=\left[a_{\zeta}-b_{\zeta}\right], \zeta \in[0,1]$, thus $[a]-[a]=[0]$; 
(iii) $[a] \cdot[b] \triangleq\left[a_{\zeta}\right] \cdot\left[b_{\zeta}\right]=\left[a_{\zeta} \cdot b_{\zeta}\right], \zeta \in[0,1]$;

(iv) $[a] \cdot([b]+[c])=[a] \cdot[b]+[a] \cdot[c]$;

(v) $[b]^{-1} \triangleq\left[b_{\zeta}\right]^{-1}=\left[b_{\zeta}^{-1}\right], \zeta \in[0,1]$ if $0 \notin[b]$, thus $[a] /[b] \triangleq[a] \cdot[b]^{-1}$.

(vi) $[a]<[b]$ if for any $\zeta \in[0,1]: a_{\zeta}<b_{\zeta}$.

It is easy to prove that the set $\mathcal{F}(\mathbb{R})$ of all continuous function $\left[x_{\zeta}\right]$ is closed with respect to operations (i)-(v).

Definition B.1. (i) Let $x \mapsto f(x): \mathbb{R} \rightarrow \mathbb{R}$ by any continuous function. Then we define the interval-valued function $f([x]): \mathcal{F}(\mathbb{R}) \rightarrow \mathcal{F}(\mathbb{R})$, which extend $f(x)$ from $\mathbb{R}$ up to $\mathcal{F}(\mathbb{R})$ by the following formula

$$
f([x])=\left[f\left(x_{\zeta}\right)\right] \in \mathcal{F}(\mathbb{R}), \zeta \in[0,1] .
$$

It is also denoted by $f\left(\left[x_{\zeta}\right]\right), \zeta \in[0,1]$. Standard interval-valued functions

$$
\sin [x] ; \cos [x] ; \tan [x] ; \arctan [x] ; \exp [x] ; \ln [x] ;[x]] ; \sqrt[n]{[x]}, \text { etc. }
$$

are well defined by Equation (B.13) in obvious way.

Let $x \mapsto f(x): \mathbb{R}^{n} \rightarrow \mathbb{R}^{n}$ by any continuous function of $n$ real variables

$$
x=\left(x_{1}, x_{2}, \cdots, x_{n}\right) .
$$

Then we define the interval-valued function $f([x]): \mathcal{F}^{n}(\mathbb{R}) \rightarrow \mathcal{F}(\mathbb{R})$, where $[x]=\left(\left[x_{1}\right],\left[x_{2}\right], \cdots,\left[x_{n}\right]\right)$, which extend $f(x)$ from $\mathbb{R}$ up to $\mathcal{F}^{n}(\mathbb{R})$ by the following formula

$$
f([x])=f\left(\left[x_{1}\right],\left[x_{2}\right], \cdots,\left[x_{n}\right]\right)=\left[f\left(x_{1, \zeta}, x_{2, \zeta}, \cdots, x_{n, \zeta}\right)\right] \in \mathcal{F}(\mathbb{R}), \zeta \in[0,1] .
$$

It is also denoted by $f\left(\left[x_{\zeta}\right]\right)=f\left(\left[x_{1, \zeta}\right],\left[x_{2, \zeta}\right], \cdots,\left[x_{n, \zeta}\right]\right), \zeta \in[0,1]$.

(ii) Let $f(x): \mathbb{R} \rightarrow \mathbb{R}$ by any continuous function and the $\mathrm{n}$-th derivative $f^{(n)}(x), x \in(-\infty,+\infty)$ exists. Then we define the $\mathrm{n}$-th derivative $f^{(n)}([x])$ of the interval-valued function $f([x])$ by the following formula

$$
f^{(n)}([x])=\left[f^{(n)}\left(x_{\zeta}\right)\right] \in \mathcal{F}(\mathbb{R}), \zeta \in[0,1] .
$$

Definition B.2. (i) Let $x \mapsto f(x): \mathbb{R} \rightarrow \mathbb{R}$ by any continuous function. Then we define the integration of interval-valued function $f([x]): \mathcal{F}(\mathbb{R}) \rightarrow \mathcal{F}(\mathbb{R})$ by the following formula

$$
\int_{[a]}^{[b]} f([x]) \mathrm{d}[x] \triangleq\left[\int_{a_{\zeta}}^{b_{\zeta}} f\left(x_{\zeta}\right) \mathrm{d} x_{\zeta}\right], \zeta \in[0,1] .
$$

(ii) Let $x \mapsto f\left(x_{1}, x_{2}, \cdots, x_{n}\right): \mathbb{R}^{n} \rightarrow \mathbb{R}$ by any continuous function. Multiple integration of the interval-valued function $f([x]): \mathcal{F}(\mathbb{R}) \rightarrow \mathcal{F}(\mathbb{R})$ function in $n$ variables

$$
\begin{aligned}
& \int_{\left[a_{1}\right]\left[a_{2}\right]}^{\left[b_{1}\right]\left[b_{2}\right]} \cdots \int_{\left[a_{n}\right]}^{\left[b_{n}\right]} f\left(\left[x_{1}\right],\left[x_{2}\right], \cdots,\left[x_{n}\right]\right) \mathrm{d}\left[x_{1}\right] \\
& \triangleq\left[\int_{a_{1, \zeta}}^{b_{1, \zeta}} \int_{a_{1, \zeta}}^{b_{2, \zeta}} \cdots \int_{a_{n, \zeta}}^{b_{n, \zeta}} f\left(x_{1, \zeta}, x_{2, \zeta}, \cdots, x_{n, \zeta}\right) \mathrm{d} x_{1, \zeta} \mathrm{d} x_{2, \zeta} \cdots \mathrm{d} x_{n, \zeta}\right], \zeta \in[0,1] .
\end{aligned}
$$

(iii) Let $\lambda \mapsto f(\lambda): \mathbb{R} \rightarrow \mathbb{R}$ by any continuous function. Then we define the 
integration of the interval-valued function $\left[f_{\zeta}(\lambda)\right]: \mathbb{R} \rightarrow \mathcal{F}(\mathbb{R})$ by the following formula

$$
\int_{\alpha}^{\beta}\left[f_{\zeta}(\lambda)\right] \mathrm{d} \lambda \triangleq\left[\int_{\alpha}^{\beta} f_{\zeta}(\lambda) \mathrm{d} \lambda\right], \zeta \in[0,1] .
$$

Remark B.3. Remind that from the second postulate of special relativity, together with homogeneity of spacetime and isotropy of space, it follows that the spacetime interval $s_{1,2}$ between two arbitrary events called $\boldsymbol{e}_{1}=\boldsymbol{e}_{1}\left(t_{1}, x_{1}, y_{1}, z_{1}\right)$ and $\boldsymbol{e}_{2}=\boldsymbol{e}_{2}\left(t_{2}, x_{2}, y_{2}, z_{2}\right)$ is

$$
s_{1,2}=\sqrt{c^{2}\left(t_{1}-t_{2}\right)^{2}-\left(x_{1}-x_{2}\right)^{2}-\left(y_{1}-y_{2}\right)^{2}-\left(z_{1}-z_{2}\right)^{2}} .
$$

Thus the square of the interval (B.19) or Minkowski's metric reads

$$
s_{1,2}=\sqrt{c^{2}\left(t_{1}-t_{2}\right)^{2}-\left(x_{1}-x_{2}\right)^{2}-\left(y_{1}-y_{2}\right)^{2}-\left(z_{1}-z_{2}\right)^{2}} \text {. }
$$

or in differential form

$$
\mathrm{d} s^{2}=c^{2} \mathrm{~d} t^{2}-\mathrm{d} x^{2}-\mathrm{d} y^{2}-\mathrm{d} z^{2} .
$$

Semi Riemannian manifold endroved with Minkowski's metric (B.21) is called Minkowski's spacetime and denoted by $M^{1,3}$.

Remark B.4.Note that Minkowski's spacetime relevant only for classical events $\boldsymbol{e}(t, x, y, z)$, i.e. the events with exactly measured coordinates: $t, x, y, z$. From consideration above it is clear that for physical events corresponding to quantum measurement the classical model based on Minkowski's geometry no longer holds.In order to avoid this difficultness we apply the pont free Minkowski's geometry [16] related to quantum measurement on inertial relativistic frame of reference.

The coordinate-independent definition of the square of the interval-valued line element $\mathrm{d}[s]$ in an n-dimensional Riemannian or Pseudo Riemannian manifold (in physics usually a Lorentzian manifold) is the "square of the length" of an interval-valued infinitesimal displacement $\mathrm{d}[\boldsymbol{q}]$ (in pseudo Riemannian manifolds possibly negative) whose square root should be used for computing the generalized, i.e. interval-valued curve length:

$$
\mathrm{d}[s]^{2}=\mathrm{d}[\boldsymbol{q}] \circ \mathrm{d}[\boldsymbol{q}]=g(\mathrm{~d}[\boldsymbol{q}], \mathrm{d}[\boldsymbol{q}]),
$$

where $g$ is the interval-valued metric tensor, o denotes inner product, and $\mathrm{d}[\boldsymbol{q}]$ an interval-valued infinitesimal displacement on the (pseudo) Riemannian manifold. By parameterising interval-valued curve $[q](\lambda) \in \mathcal{F}^{n}(\mathbb{R})$ parametrised by a parameter $\lambda \in \mathbb{R}$, we can define the arc length of the curve length of the curve between $[q]\left(\lambda_{1}\right) \in \mathcal{F}^{n}(\mathbb{R})$, and $[q]\left(\lambda_{2}\right) \in \mathcal{F}^{n}(\mathbb{R})$ is the interval-valued integral

$$
\begin{aligned}
{[s] } & =\int_{\lambda_{1}}^{\lambda_{2}} \mathrm{~d} \lambda \sqrt{\left|\mathrm{d}[s]^{2}\right|}=\int_{\lambda_{1}}^{\lambda_{2}} \mathrm{~d} \lambda \sqrt{\left|g\left(\frac{\mathrm{d}[q](\lambda)}{\mathrm{d} \lambda}, \frac{\mathrm{d}[q](\lambda)}{\mathrm{d} \lambda}\right)\right|} \\
& =\int_{\lambda_{1}}^{\lambda_{2}} \mathrm{~d} \lambda \sqrt{\mid g_{i j}\left(\frac{\mathrm{d}\left[q^{i}\right](\lambda)}{\mathrm{d} \lambda}, \frac{\mathrm{d}\left[q^{j}\right](\lambda)}{\mathrm{d} \lambda}\right) .}
\end{aligned}
$$


The square of arc length $\mathrm{d}[\mathrm{s}]^{2}$ with the metric is even more easy to see in $n$-dimensional general interval-valued curvilinear coordinates $[q]=\left(\left[q_{1}\right],\left[q_{2}\right],\left[q_{3}\right], \cdots,\left[q_{n}\right]\right)$, where it is written as a symmetric rank 2 tensor coinciding with the metric tensor:

$$
\mathrm{d}[s]^{2}=\left[g_{i j}\right] \mathrm{d}\left[q^{i}\right] \mathrm{d}\left[q^{j}\right]=[g] .
$$

Here the indices $i$ and $j$ take values $1,2,3, \cdots, n$ and Einstein summation convention is used. Common examples of (pseudo) Riemannian point-free spaces include three-dimensional point free space (no inclusion of time coordinates), and indeed four-dimensional point-free spacetime.

Following are importent examples of how the interval-valued line elements are found from the interval-valued metric.

\section{Interval-valued Cartesian coordinates.}

The simplest interval-valued line element is in interval-valued Cartesian coordinates-in which case the metric is just the usual Kronecker delta

$$
\left[g_{i j}\right]=\left[\delta_{i j}\right] \equiv \delta_{i j} \text {. }
$$

The general interval-valued curvilinear coordinates reduce to interval-valued Cartesian coordinates: $\left(\left[q^{1}\right],\left[q^{2}\right],\left[q^{3}\right]\right)=([x],[y],[z])$ so

$$
\mathrm{d}[s]^{2}=\left[g_{i j}\right] \mathrm{d}\left[q^{i}\right] \mathrm{d}\left[q^{j}\right]=\mathrm{d}[x]^{2}+\mathrm{d}[y]^{2}+\mathrm{d}[z]^{2} .
$$

\section{Interval-valued cylindrical coordinates.}

For the conversion between interval-valued cylindrical and interval-valued Cartesian coordinates, it is convenient to assume that the reference plane of the former is the Cartesian $[x][y]$-plane (with equation $[z]=0$ ), and the cylindrical axis is the interval-valued Cartesian $[z]$-axis. Then the interval-valued $[z]$-coordinate is the same in both systems, and the correspondence between interval-valued cylindrical $([\rho],[\varphi],[z])$ and interval-valued Cartesian $([x],[y],[z])$ are the same as for interval-valued polar coordinates, namely

$$
\begin{aligned}
& {[x]=[\rho] \cdot \cos [\varphi],[y]=[\rho] \cdot \sin [\varphi],[\rho]=\sqrt{[x]^{2}+[y]^{2}},} \\
& {[z]=[z],}
\end{aligned}
$$

where

$$
[\varphi]= \begin{cases}{[0]} & \text { if }[x]=0 \text { and }[y]=0 \\ \arcsin \left(\frac{[y]}{[\rho]}\right) & \text { if }[x] \geq 0 \\ \arctan \left(\frac{[y]}{[x]}\right) & \text { if }[x]>0 \\ -\arcsin \left(\frac{[y]}{[\rho]}\right)+[\pi] & \text { if }[x]<0\end{cases}
$$

The line element is:

$$
\mathrm{d}[s]^{2}=\mathrm{d}[r]^{2}+[r]^{2} \mathrm{~d}[\varphi]^{2}+\mathrm{d}[z]^{2} .
$$




\section{Point free Minkowskian spacetime.}

The interval-valued Minkowski metric is:

$$
\left(\left[g_{i j}\right]\right)=\left(\begin{array}{cccc}
{[1]} & {[0]} & {[0]} & {[0]} \\
{[0]} & -[1] & {[0]} & {[0]} \\
{[0]} & {[0]} & -[1] & {[0]} \\
{[0]} & {[0]} & {[0]} & -[1]
\end{array}\right)
$$

where one sign or the other is chosen, both conventions are used. This applies only for flat point free spacetime. The interval-valued coordinates are given by the interval-valued 4-position:

$$
[\boldsymbol{x}]=([t],[x],[y],[z]),
$$

so the interval-valued line element is:

$$
\mathrm{d}[s]^{2}=c^{2} \mathrm{~d}[t]^{2}-\mathrm{d}[x]^{2}-\mathrm{d}[y]^{2}-\mathrm{d}[z]^{2} .
$$

in Thus in interval-valued polar coordinates interval-valued line element (B.32) reads

$$
\mathrm{d}[s]^{2}=c^{2} \mathrm{~d}[t]^{2}-\mathrm{d}[r]^{2}-[r]^{2} \mathrm{~d}[\varphi]^{2}-\mathrm{d}[z]^{2} .
$$

The transformation to a non inertial frame of reference $\left(\left[t^{\prime}\right],\left[r^{\prime}\right],\left[\varphi^{\prime}\right],\left[z^{\prime}\right]\right)$ rotating at the uniform angular rate $\omega$ with respect to the starting inertial frame (B.33) is given by

$$
[t]=\left[t^{\prime}\right],[r]=\left[r^{\prime}\right],\left[\varphi^{\prime}\right]=[\varphi]-\omega[t] .
$$

Thus, interval-valued line element given by the Equation (B.33) by the transformation (B.34) becomes the following interval-valued line element (interval-valued Langevin metric) in rotating frame reads

$$
\begin{aligned}
\mathrm{d}[s]^{2}= & \left(1-\frac{[r]^{2} \omega^{2}}{c^{2}}\right) c^{2} \mathrm{~d}[t]^{2}-2 \omega[r]^{2} \mathrm{~d}\left[\varphi^{\prime}\right] \mathrm{d}[t] \\
& -\mathrm{d}[r]^{2}-[r]^{2} \mathrm{~d}\left[\varphi^{\prime}\right]^{2}-\mathrm{d}[z]^{2} .
\end{aligned}
$$

\section{Appendix C. The Interval-Valued Trajectories Corresponding to 2-Dimensional Wave Packet}

I. The interval-valued trajectories corresponding to 2-dimensional wave packet which propagates in inertial frame of references.

Assuming that $\alpha_{2} \leq \varphi \leq \alpha_{1}, \alpha_{2} \leq \theta \leq \alpha_{1}$ (see Figure 1), $[t]=[\underline{t}, \bar{t}], \underline{t} \leq t_{\zeta}^{\prime} \leq \bar{t}, \quad[r]=[\underline{r}, \bar{r}], \underline{r} \leq r_{\zeta}^{\prime} \leq \bar{r},[\varphi]=\left[\alpha_{2}, \alpha_{1}\right],[\theta]=\left[\alpha_{2}, \alpha_{1}\right]$, see Remark B.2, from Equation (A.22) and Equation (B.11)-Equation (B.12) we obtain

$$
P_{I F}([t],[r],[\varphi],[\theta])=\left[\int_{\underline{t}}^{t_{\zeta}^{\prime}} \int_{\alpha_{2}}^{\alpha_{1}} w_{I F}\left(r_{\zeta}, \varphi_{\zeta}, \theta_{\zeta}, t_{\zeta}\right) \mathrm{d} t_{\zeta} \mathrm{d} \varphi_{\zeta}\right]
$$

Thus $P_{I F}([t],[r],[\varphi],[\theta])$ is the interval-valued probability of detecting a "click" in inertial frame of references at interval-valued instant $[t]=[\underline{t}, \bar{t}]$ at an "point" 


$$
([r],[\varphi]) \in \mathcal{F}(\mathbb{R}) \times \mathcal{F}\left(\left[\alpha_{2}, \alpha_{1}\right]\right)
$$

with interval-valued coordinates $[r]=[\underline{r}, \bar{r}]$ and $[\varphi]=\left[\alpha_{2}, \alpha_{1}\right]$. Here the probability density $w_{I F}(r, \varphi, \theta, t)$ is given in accordance with Equation (A.19) reads

$$
w_{I F}(r, \varphi, \theta, t) \sim \alpha^{-1} \Gamma H\left(t-\frac{r \cos \psi}{c}\right) \exp \left[-\Gamma\left(t-\frac{r \cos \psi}{c}\right)\right],
$$

where $\psi=\varphi-\theta, \alpha=\alpha_{1}-\alpha_{2}$ is angular size of the detector (see Figure 2).

Remark C.1. Note that normalizing factor in Equation (C.3) necessarily contains a multiplier $\alpha^{-1}$, since $\psi \ll 2 \pi \mathrm{rad}$ and thus $\cos \psi \simeq 1$. Therefore the conditions is given by Equation (B.5) and (B.6) are satisfied, since

$$
P_{I F}\left([t],[r],[\varphi],\left[\theta^{\prime}\right]\right) \simeq\left[\int_{\underline{t}}^{t_{\zeta}^{\prime}} \int_{\alpha_{2}^{\prime}}^{\alpha_{1}^{\prime}} w_{I F}\left(r_{\zeta}, \varphi_{\zeta}, \theta_{\zeta}, t_{\zeta}\right) \mathrm{d} t_{\zeta} \mathrm{d} \varphi_{\zeta}\right]<1 / 2,
$$

where $\left[\alpha_{2}^{\prime}, \alpha_{1}^{\prime}\right] \varsubsetneqq 0.5\left[\alpha_{2}, \alpha_{1}\right]$ and

$$
\left[\int_{\underline{t}}^{t_{\zeta}} \int_{[0,2 \pi]\left\lceil\alpha_{2}, \alpha_{1}\right]} w_{I F}\left(r_{\zeta}, \varphi_{\zeta}, \theta_{\zeta}, t_{\zeta}\right) \mathrm{d} t_{\zeta} \mathrm{d} \varphi_{\zeta}\right]=0 .
$$

Substituting Equation (C.3) into Equation (C.1) we get

$$
\begin{aligned}
& {\left[P_{I F}([t],[r],[\varphi],[\theta])\right]} \\
& \sim\left[\int_{\underline{t}}^{t_{\zeta}^{\prime}} \int_{\alpha_{2}}^{\alpha_{1}} \alpha^{-1} \Gamma H\left(t_{\zeta}-\frac{r_{\zeta} \cos \left(\varphi_{\zeta}-\theta_{\zeta}\right)}{c}\right)\right. \\
& \left.\quad \times \exp \left[-\Gamma\left(t_{\zeta}-\frac{r_{\zeta} \cos \left(\varphi_{\zeta}-\theta_{\zeta}\right)}{c}\right)\right] \mathrm{d} t_{\zeta} \mathrm{d} \varphi_{\zeta}\right],
\end{aligned}
$$

where $\zeta \in[0,1]$. Note that the condition is given by Equation (B.11) now reads

$$
\left[P_{I F}([t],[r],[\varphi],[\theta])\right] \simeq 1 .
$$

From Equation (C.6) and Equation (C.7) and by the definition of the Heaviside step function $H\left(\tau_{\zeta}\right)$, where $\tau_{\zeta}=t_{\zeta}-\frac{r_{\zeta} \cos \left(\varphi_{\zeta}-\theta_{\zeta}\right)}{c}$, we get

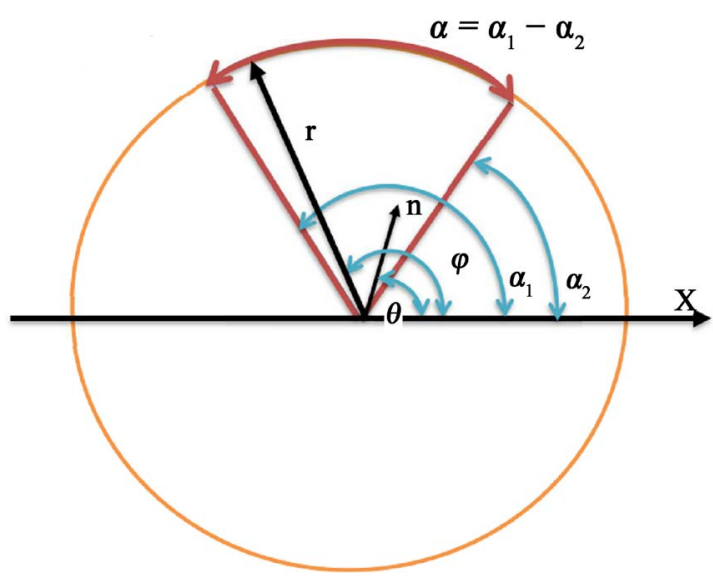

Figure 2. Quantum measurements on inertial frame of reference. $\alpha_{1} \leq \varphi \leq \alpha_{2}$ a is angular size of detector: $\alpha=\alpha_{1}-\alpha_{2}\left[\psi_{*}\right]=\left[\underline{\psi}_{*}, \bar{\psi}_{*}\right]=[0, \alpha] \ll 2 \pi \operatorname{rad}$. 


$$
[t]-\frac{[r] \cos ([\varphi]-[\theta])}{c}=[t]-\frac{[r] \cos ([\psi])}{c} \geq 0,
$$

where $\psi=\varphi-\theta$, (see Figure 2). Thus on inertial relativistic frame of reference endroved with interval-valued polar coordinates (B.27) photodetection signal propagate with a probability $\simeq 1$, in accordance with the following interval-valued law

$$
[r] \simeq c_{[\psi]}[t],[\varphi]=\left[\alpha_{2}, \alpha_{1}\right]
$$

where

$$
c_{[\psi]} \simeq \frac{c}{\cos ([\psi])},
$$

and where $[\psi]=\left[0, \alpha_{1}-\alpha_{2}\right]$, (see Figure 2).

II. The interval-valued trajectories corresponding to 2-dimensional wave packet which propagates in rotating non inertial frame of references.

Remark C.2. Note that the probability density $w_{I F}(r, \varphi, \theta, t)$ in inertial frame given by Equation (C.3) that is scalar quantity and therefore corresponding probability density $w_{R F}(r, \varphi, \theta, t)$ in rotating non inertial frame endrowed with canonical Langevin metric reads

$$
w_{R F}(r, \varphi, \theta, t) \sim \alpha^{-1} \Gamma H\left(t-\frac{r \cos (\psi-\omega t)}{c}\right) \exp \left[-\Gamma\left(t-\frac{r \cos (\psi-\omega t)}{c}\right)\right],
$$

where $\psi=\varphi-\theta$, (see Figure 3 ).

Remark C.3. Note that in the rotating frame the angular size of the detector is not exactly the same as in the frame at rest and equal [29]: $\alpha^{*}=\alpha / \sqrt{1-\frac{\omega^{2} \bar{r}^{2}}{c^{2}}} \simeq \alpha$.

Assuming that $\alpha_{2} \leq \varphi \leq \alpha_{1}, \alpha_{2} \leq \theta \leq \alpha_{1}$ (see Figure 3), $[t]=[\underline{t}, \bar{t}], \underline{t} \leq t_{\zeta}^{\prime} \leq \bar{t}$, $[r]=[\underline{r}, \bar{r}], \underline{r} \leq r_{\zeta}^{\prime} \leq \bar{r},[\varphi]=\left[\alpha_{2}, \alpha_{1}\right],[\theta]=\left[\alpha_{2}, \alpha_{1}\right]$, see Remark B.2, from Equation (A.6) and Equation (B.11)-Equation (B.12) we obtain

$$
P_{R F}([t],[r],[\varphi],[\theta-\omega t])=\left[\int_{\underline{t}}^{t_{\zeta}^{\prime}} \int_{\alpha_{2}^{*}}^{\alpha_{1}^{*}} w_{R F}\left(r_{\zeta}, \varphi_{\zeta}, \theta_{\zeta}-\omega t, t_{\zeta}\right) \mathrm{d} t_{\zeta} \mathrm{d} \varphi_{\zeta}\right]
$$

where $\alpha_{1}^{*}=\alpha / \sqrt{1-\frac{\omega^{2} \bar{r}^{2}}{c^{2}}} \simeq \alpha_{1}, \alpha_{2}^{*}=\alpha / \sqrt{1-\frac{\omega^{2} \bar{r}^{2}}{c^{2}}} \simeq \alpha_{2}$.

Thus $P_{R F}([t],[r],[\varphi],[\theta])$ is the interval-valued probability of detecting a "click" in non inertial frame of references at interval-valued instant $[t]=[\underline{t}, \bar{t}]$ at an "point"

$$
([r],[\varphi]) \in \mathcal{F}(\mathbb{R}) \times \mathcal{F}\left(\left[\alpha_{2}^{*}, \alpha_{1}^{*}\right]\right)
$$

Substituting Equation (C.11) into Equation (C.12) we get

$$
\begin{aligned}
& {\left[P_{R F}([t],[r],[\varphi],[\theta-\omega t])\right]} \\
& \sim\left[\int_{\underline{t}}^{t_{\zeta}^{\prime}} \int_{\alpha_{2}^{*}}^{\alpha_{1}^{*}} \alpha^{-1} \Gamma H\left(t_{\zeta}-\frac{r_{\zeta} \cos \left(\varphi_{\zeta}-\theta_{\zeta}-\omega t\right)}{c}\right)\right. \\
& \left.\quad \times \exp \left[-\Gamma\left(t_{\zeta}-\frac{r_{\zeta} \cos \left(\varphi_{\zeta}-\theta_{\zeta}-\omega t\right)}{c}\right)\right] \mathrm{d} t_{\zeta} \mathrm{d} \varphi_{\zeta}\right],
\end{aligned}
$$


where $\zeta \in[0,1]$. Note that the condition is given by Equation (B.11) now reads

$$
\left[P_{R F}([t],[r],[\varphi],[\theta-\omega t])\right] \simeq 1 .
$$

From Equation (C.14) and Equation (C.15) and by the definition of the Heaviside step function $H\left(\tau_{\zeta}\right)$, where $\tau_{\zeta}=t_{\zeta}-\frac{r_{\zeta} \cos \left(\varphi_{\zeta}-\theta_{\zeta}\right)}{c}$, we get

$$
[t]-\frac{[r] \cos \left(\left[\varphi^{*}\right]-\left[\theta^{*}\right]-\omega t\right)}{c}=[t]-\frac{[r] \cos \left(\left[\psi^{*}\right]-\omega t\right)}{c} \geq 0,
$$

where $\varphi^{*}=\varphi / \sqrt{1-\frac{\omega^{2} \bar{r}^{2}}{c^{2}}} \simeq \varphi, \theta^{*}=\theta / \sqrt{1-\frac{\omega^{2} \bar{r}^{2}}{c^{2}}} \simeq \theta$ and $\psi^{*}=\varphi^{*}-\theta^{*}$, (see Figure 3).Thus on non inertial relativistic frame of reference endroved with interval-valued polar coordinates (B.27) photodetection signal propagate with a probability $\simeq 1$, in accordance with the following interval-valued law

$$
[r] \simeq c_{\left[\psi^{*}-\omega t\right]}[t]
$$

and

$$
\left[\varphi^{*}\right]=\left[\alpha_{2}, \alpha_{1}\right] / \sqrt{1-\frac{\omega^{2} \bar{r}^{2}}{c^{2}}}=\left[\alpha_{2}^{*}, \alpha_{1}^{*}\right]
$$

where

$$
C_{\left[\psi^{*}\right]} \simeq \frac{c}{\cos \left(\left[\psi^{*}-\omega t\right]\right)}
$$

and

$$
\begin{aligned}
{\left[\psi_{*}\right] } & =\left[\underline{\psi}_{*}, \bar{\psi}_{*}\right]=\left[0, \alpha^{*}\right]=\left[0,\left(\alpha_{1}-\alpha_{2}\right) / \sqrt{1-\frac{\omega^{2} \bar{r}^{2}}{c^{2}}}\right] \\
& \simeq[0, \alpha]=\left[0, \alpha_{1}-\alpha_{2}\right] \ll 2 \pi \mathrm{rad} .
\end{aligned}
$$

\section{Appendix D. Quantum Measurement on Non Inertial Relativistic Frame of Reference. Point Free Lorentzian Geometry Corresponding to Mössbauer Rotor Experiment}

Let $\breve{M}^{1,3}$ be point free Minkowskian space-time endroved with the following interval-valued line element in interval-valued polar coordinates

$$
\mathrm{d}[s]^{2}=c_{[\psi]}^{2} \mathrm{~d}[t]^{2}-\mathrm{d}[r]^{2}-[r]^{2} \mathrm{~d}[\varphi]^{2}-\mathrm{d}[z]^{2},
$$

where $\psi=\varphi-\theta$, (see Figure 1 ).

Remark D.1. Note that the interval-valued line element (D.1) corresponding to photodetection signal which propagate with a probability $\simeq 1$ in accordance with the interval-valued law is given by Equation (C.9)-Equation (C.10).

The transformation to a non inertial frame of reference $\left(\left[t^{\prime}\right],\left[r^{\prime}\right],\left[\varphi^{\prime}\right],\left[z^{\prime}\right]\right)$ rotating at the uniform angular rate $\omega$ with respect to the starting inertial frame (D.1) is given by 


$$
[t]=\left[t^{\prime}\right],[r]=\left[r^{\prime}\right],\left[\varphi^{\prime}\right]=[\varphi]-\omega[t] .
$$

Remark D.2. Note that below we abbraviate $\varphi$ instead $\varphi^{\prime}$ but this should not introduce the misleading.

Thus, interval-valued line element given by the Equation (D.1) by the transformation (D.2) becomes the following interval-valued line element (interval-valued Langevin metric) in rotating frame reads

$$
\begin{aligned}
\mathrm{d}[s]^{2}= & \left(1-\frac{[r]^{2} \omega^{2}}{c_{[\psi]-\omega t}^{2}}\right)\left(c_{[\psi]-\omega t}^{2}\right) \mathrm{d}[t]^{2}-2 \omega[r]^{2} \mathrm{~d}[\varphi] \mathrm{d}[t] \\
& -\mathrm{d}[r]^{2}-[r]^{2} \mathrm{~d}[\varphi]^{2}-\mathrm{d}[z]^{2} .
\end{aligned}
$$

For simplicity but without loss of generality, we consider now 2-dimensional interval-valued Langevin metric (D.5) (see Figure 3):

$$
\mathrm{d}[s]^{2}=\left(1-\frac{[r]^{2} \omega^{2}}{c_{[\psi]-\omega t}^{2}}\right)\left(c_{[\psi]-\omega t}^{2}\right) \mathrm{d}[t]^{2}-2 \omega[r]^{2} \mathrm{~d}[\varphi] \mathrm{d}[t]-\mathrm{d}[r]^{2}-[r]^{2} \mathrm{~d}[\varphi]^{2},
$$

By substituting $\left[\varphi_{*}\right]:=\left[\underline{\varphi}_{*}, \bar{\varphi}_{*}\right]=\left[\alpha_{1}^{*}, \alpha_{2}^{*}\right]=$ const and $\left[\psi_{*}\right]=\left[\underline{\psi}_{*}, \bar{\psi}_{*}\right]=\left[0, \alpha^{*}\right]$ (see Remark C.3) into Equation (D.4) we get

$$
\mathrm{d}[s]^{2}=\left(1-\frac{[r]^{2} \omega^{2}}{c_{\left[\psi_{*}\right]-\omega t}^{2}}\right)\left(c_{\left[\psi_{*}\right]-\omega t}^{2}\right) \mathrm{d}[t]^{2}-\mathrm{d}[r]^{2}=0 .
$$

From Equation (D.5) we obtain

$$
\frac{\mathrm{d}[r]}{\sqrt{1-\frac{[r]^{2} \omega^{2}}{c_{\left[\psi_{*}\right]-\omega t}^{2}}}}=\left(c_{\left[\psi_{*}\right]-\omega t}\right) \mathrm{d}[t] .
$$

Note that $\omega t \ll 2 \pi$ rad and therefore from Equation (D.6) and Equation (C.10) we obtain

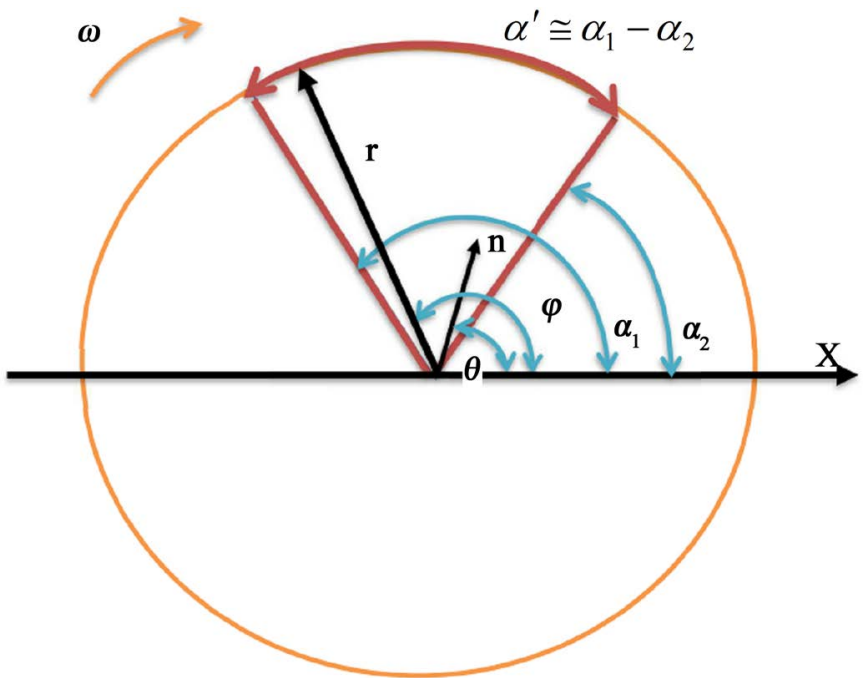

Figure 3. Mössbauer rotor experiment. Quantum measurements on non inertial frame of reference. $\varphi^{\prime}=\varphi-\omega t ; \alpha_{1} \leq \varphi \leq \alpha_{2} \quad \alpha^{\prime} \simeq \alpha_{1}-\alpha_{2},\left[\alpha^{\prime}\right] \simeq\left[0, \alpha_{1}-\alpha_{2}\right]$. 


$$
\frac{\mathrm{d}[r]}{\sqrt{1-\frac{[r]^{2} \omega^{2}}{c_{\left[\psi_{*}\right]}^{2}}}}=\left(c_{[\psi]}\right) \mathrm{d}[t] .
$$

We rewrite now Equation (D.7) in the following equivalent form

$$
\left[\frac{\mathrm{d} r_{\zeta}}{\sqrt{1-\frac{r_{\zeta}^{2} \omega^{2}}{c_{\left[\psi_{*}\right]}^{2}}}}\right]=\left(c_{\left[\psi_{*}\right]}\right)\left[\mathrm{d} t_{\zeta}\right] .
$$

By integrating from Equation (D.7) we get

$$
\left[\int_{\underline{r}}^{\bar{r}} \frac{\mathrm{d} r_{\zeta}}{\sqrt{1-\frac{r_{\zeta}^{2} \omega^{2}}{c_{[\psi]}^{2}}}}\right]=\left(c_{[\psi]}\right)\left[\int_{\underline{t}}^{\bar{t}} \mathrm{~d} t_{\zeta}^{\prime}\right] .
$$

From Equation (D.9) we get

$$
\frac{c_{[\psi]}}{\omega} \arcsin \left(\frac{\omega[r]}{c_{\left[\psi_{*}\right]}}\right)=\left(c_{\left[\psi_{*}\right]}\right)\left(\left[t_{2}\right]-\left[t_{1}\right]\right) .
$$

From Equation (D.10) we obtain

$$
\frac{c_{\left[\psi_{*}\right]}}{\omega}\left[\frac{\omega[r]}{c_{\left[\psi_{*}\right]}}+\frac{1}{6}\left(\frac{\omega[r]}{c_{\left[\psi_{*}\right]}}\right)^{3}\right] \simeq\left(c_{\left[\psi_{*}\right]}\right)\left(\left[t_{2}\right]-\left[t_{1}\right]\right) .
$$

From Equation (D.11) and Equation (C.10) we get

$$
[r]\left[1+\frac{1}{6}\left(\frac{\omega[r]}{c_{\left[\psi_{*}\right]}}\right)^{3}\right] \simeq \frac{c\left(\left[t_{2}\right]-\left[t_{1}\right]\right)}{\cos ([\psi])} .
$$

From Equation (D.11) we get

$$
\frac{[r]}{c}\left[1+\frac{1}{6}\left(\frac{\omega[r]}{c_{\left[\psi_{*}\right]}}\right)^{3}\right] \cos \left(\left[\psi_{*}\right]\right) \simeq\left[t_{2}\right]-\left[t_{1}\right] .
$$

From Equation (D.13) finally we get in appropriate approximation

$$
\frac{\bar{r}}{c}\left[1+\frac{1}{6}\left(\frac{\omega \bar{r}}{c}\right)^{2}\right] \simeq\left(\bar{t}_{2}-\bar{t}_{1}\right) .
$$

since $\cos \left(\left[\psi_{*}\right]\right) \simeq 1$.

Remark D.3. Note that Equation (D.14) is obtained without any references to notion of the classical trajectories.

Remark D.4. Note that Equation (D.14) coincides with Equation (16) from [2]. We rewrite now Equation (D.14) in the following form 


$$
t_{I F} \simeq t_{R F}\left[1+\frac{1}{6}\left(\frac{\omega r}{c}\right)^{2}\right]^{-1} \simeq t_{R F}\left[1-\frac{1}{6}\left(\frac{\omega r}{c}\right)^{2}\right],
$$

where: (i) $t_{I F}$ is propagation time of the photodetection signal measured by observer on inertial frame of reference and (ii) $t_{R F}$ is propagation time of the photodetection signal measured by observer on non inertial (rotating) frame of reference.

From Equation (A.19) using the transformation is given by Equation (D.2) we obtain

$$
\begin{aligned}
& w_{R F}\left(r, \varphi-\omega t_{I F}, \theta, t_{I F}\right) \\
& \sim \alpha^{-1} \Gamma H\left(t_{I F}-\frac{r \cos \left(\psi-\omega t_{I F}\right)}{c}\right) \exp \left[-\Gamma\left(t_{I F}-\frac{r \cos \left(\psi-\omega t_{I F}\right)}{c}\right)\right],
\end{aligned}
$$

since the quantity $w(r, \varphi, \theta, t)$ is a scalar.

By substituting Equation (D.15) into Equation (D.16) we obtain

$$
\begin{aligned}
& w_{R F}\left(r, \varphi-\omega t_{R F}, \theta, t_{R F}\right) \\
& \sim \alpha^{-1} \gamma_{r} \Gamma H\left(t_{R F} \gamma_{r}-\frac{r \cos \left(\psi-\omega t_{R F} \gamma_{r}\right)}{c}\right) \\
& \quad \times \exp \left[-\Gamma\left(t_{R F} \gamma_{r}-\frac{r \cos \left(\psi-\omega t_{R F} \gamma_{r}\right)}{c}\right)\right],
\end{aligned}
$$

where

$$
\gamma_{r}=\left[1-\frac{1}{6}\left(\frac{\omega r}{c}\right)^{2}\right]
$$

From Equation (D.17) finally we obtain

$$
\begin{aligned}
& w_{R F}\left(r, \varphi-\omega t_{R F}, \theta, t_{R F}\right) \\
& \sim \alpha^{-1} \gamma_{r} \Gamma H\left(t_{R F}-\frac{r \cos \left(\psi-\omega t_{R F} \gamma_{r}\right)}{c \gamma_{r}}\right) \exp \left[-\Gamma \gamma_{r}\left(t_{R F}-\frac{r \cos \left(\psi-\omega t_{R F} \gamma_{r}\right)}{c \gamma_{r}}\right)\right] \\
& =\alpha^{-1} \gamma_{r} \Gamma H\left(t_{R F}-\frac{r \cos \left(\psi-\omega t_{R F} \gamma_{r}\right)}{c_{\|r\|}}\right) \exp \left[-\Gamma \gamma_{r}\left(t_{R F}-\frac{r \cos \left(\psi-\omega t_{R F} \gamma_{r}\right)}{c_{\|r\|}}\right)\right],
\end{aligned}
$$

where $c_{\|r\|}=c \gamma_{r},\|\boldsymbol{r}\|=r$. 